Erschienen in: Franziska Preuss et al. (2020): Nucleotide Binding, Evolutionary Insights, and Interaction Partners of the Pseudokinase Unc-51-like Kinase 4. Structure, 28, 1184-1196.e6. https://doi.org/10.1016/j.str.2020.07.016

(C) 2020. This manuscript version is made available under the CC-BY-NC-ND 4.0 license http:// creativecommons.org/licenses/by-nc-nd/4.0/

\title{
Nucleotide Binding, Evolutionary Insights, and Interaction Partners of the Pseudokinase Unc-51- like Kinase 4
}

\section{Graphical Abstract}

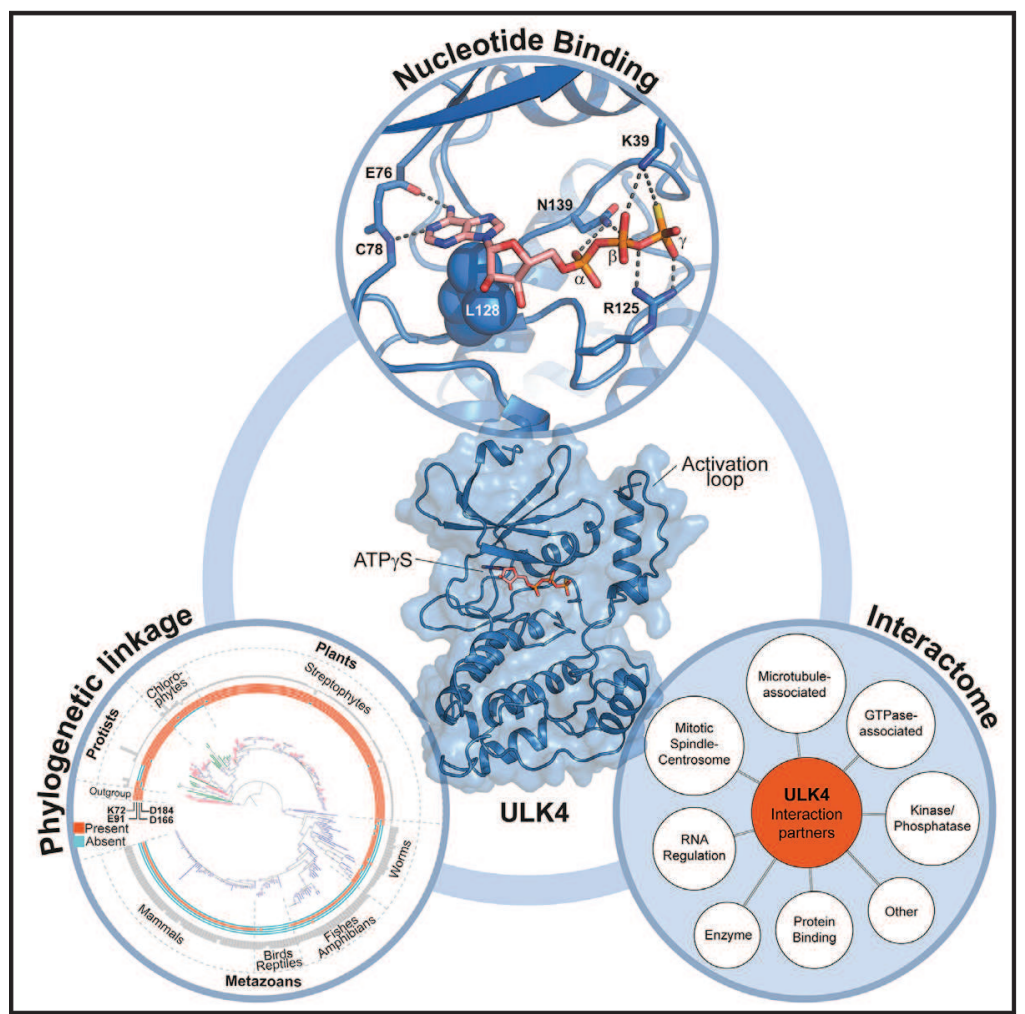

\section{Authors}

Franziska Preuss, Deep Chatterjee, Sebastian Mathea, ..., Brian Raught, Robert Rottapel, Stefan Knapp

\section{Correspondence}

knapp@pharmchem.uni-frankfurt.de

\section{In Brief}

A high-resolution structure of the understudied pseudokinase ULK4 binding ATP $\gamma \mathrm{S}$ in an unusual manner is presented by Preuss et al. Evolutionarily, the loss of canonical motifs was compensated by the co-emerging of additional structural elements. Cellular interaction studies imply a centrosomal and tubulin-associated role of ULK4.
Highlights

- Structure of the ULK4 ATP complex reveals a unique ATP binding mode

- Disease-associated mutations modulate ATP binding and ULK4 stability

- Loss of canonical motifs co-occurred in evolution with a specific activation loop

- BiolD suggests a role of ULK4 regulating centrosomal and cytoskeletal functions 


\title{
Nucleotide Binding, Evolutionary Insights, and Interaction Partners of the Pseudokinase Unc-51-like Kinase 4
}

\author{
Franziska Preuss, ${ }^{1,2,8}$ Deep Chatterjee, ${ }^{1,2,8}$ Sebastian Mathea, ${ }^{1,2}$ Safal Shrestha, ${ }^{3}$ Jonathan St-Germain, ${ }^{5}$ Manipa Saha, ${ }^{5}$ \\ Natarajan Kannan, ${ }^{3}$ Brian Raught, ${ }^{5}$ Robert Rottapel, ${ }^{5,6,7}$ and Stefan Knapp ${ }^{1,2,4,9, *}$ \\ ${ }^{1}$ Institute for Pharmaceutical Chemistry, Johann Wolfgang Goethe-University, Max-von-Laue-Str. 9, 60438 Frankfurt am Main, Germany \\ ${ }^{2}$ Buchmann Institute for Molecular Life Sciences, Structural Genomics Consortium, Johann Wolfgang Goethe-University, Max-von-Laue-Str. \\ 15, 60438 Frankfurt am Main, Germany \\ ${ }^{3}$ Institute of Bioinformatics \& Department of Biochemistry and Molecular Biology, University of Georgia, 120 Green Street, Athens, GA \\ 30602-7229, USA \\ ${ }^{4}$ German Cancer Consortium (DKTK) and Frankfurt Cancer Institute (FCl), 60596 Frankfurt am Main, Germany \\ 5Princess Margaret Cancer Centre, University Health Network, Toronto M5G 2C4, Canada \\ ${ }^{6}$ Departments of Medicine, Immunology and Medical Biophysics, University of Toronto, Toronto M5G 1L7, Canada \\ ${ }^{7}$ Division of Rheumatology, St. Michael's Hospital, Toronto M5B 1W8, Canada \\ 8 These authors contributed equally \\ 'Lead Contact \\ ${ }^{*}$ Correspondence: knapp@pharmchem.uni-frankfurt.de \\ https://doi.org/10.1016/j.str.2020.07.016
}

\section{SUMMARY}

Unc-51-like kinase 4 (ULK4) is a pseudokinase that has been linked to the development of several diseases. Even though sequence motifs required for ATP binding in kinases are lacking, ULK4 still tightly binds ATP and the presence of the co-factor is required for structural stability of ULK4. Here, we present a high-resolution structure of a ULK4-ATP $\gamma \mathrm{S}$ complex revealing a highly unusual ATP binding mode in which the lack of the canonical VAIK motif lysine is compensated by K39, located $\mathrm{N}$-terminal to $\alpha \mathrm{C}$. Evolutionary analysis suggests that degradation of active site motifs in metazoan ULK4 has co-occurred with an ULK4-specific activation loop, which stabilizes the $C$ helix. In addition, cellular interaction studies using BioID and biochemical validation data revealed high confidence interactors of the pseudokinase and armadillo repeat domains. Many of the identified ULK4 interaction partners were centrosomal and tubulin-associated proteins and several active kinases suggesting interesting regulatory roles for ULK4.

\section{INTRODUCTION}

The human genome encodes about 520 protein kinases. Structural studies revealed that protein kinases share a common topology, the canonical "kinase fold" and large-scale sequence comparison have revealed highly conserved amino acid motifs important for kinase catalytic function, including (1) the glycine-rich loop, (2) the VAIK motif containing a lysine to bridge the $\beta 3$ strand to the $\alpha \mathrm{C}$ helix in active kinases coordinating the $\mathrm{Mg}^{2+} /$ triphosphate moiety of the ATP co-factor, (3) the HRD motif with the catalytically indispensable catalytic base, (4) a conserved asparagine prior to the $\beta 7$ strand that orients the ATP phosphates, and finally (5) the DFG motif at the $\mathrm{N}$ terminus of the activation loop that is important for binding of the phosphate moieties of ATP and $\mathrm{Mg}^{2+}$ (Manning et al., 2002). However, about $10 \%$ of kinases lack one or more of these conserved motifs, which often renders them catalytically inactive or with significantly reduced catalytic activity (Boudeau et al., 2006; Kwon et al., 2019). These kinases are therefore referred to as pseudo- kinases. Instead of catalyzing the phosphoryl transfer reaction, their physiological role mediating cellular signaling is scaffolding and to serve as allosteric regulators of active enzymes (recently reviewed by Jacobsen and Murphy, 2017).

Pseudokinases are present in all major groups of the human kinome and across diverse species (Kwon et al., 2019). The structural features of pseudokinases are accordingly diverse, sharing many regulatory mechanisms observed in canonical kinases (Ha and Boggon, 2018; Jura et al., 2009; Patel et al., 2017; Scheeff et al., 2009; Shrestha et al., 2020; Zeqiraj et al., 2009b). Interestingly, many human pseudokinases have completely lost the ability to interact with nucleotides, while others still bind to and are stabilized by ATP interaction (Murphy et al., 2014). The subgroup of ATP binding pseudokinases are thought to fulfill their physiological tasks in the nucleotide-bound form. There are several variations of how pseudokinases exert their signaling roles (Murphy et al., 2017). For instance, the transmembrane growth factor receptor HER3 regulates the mitogenactivated protein kinase (MAPK) and phosphatidylinositol 3- 


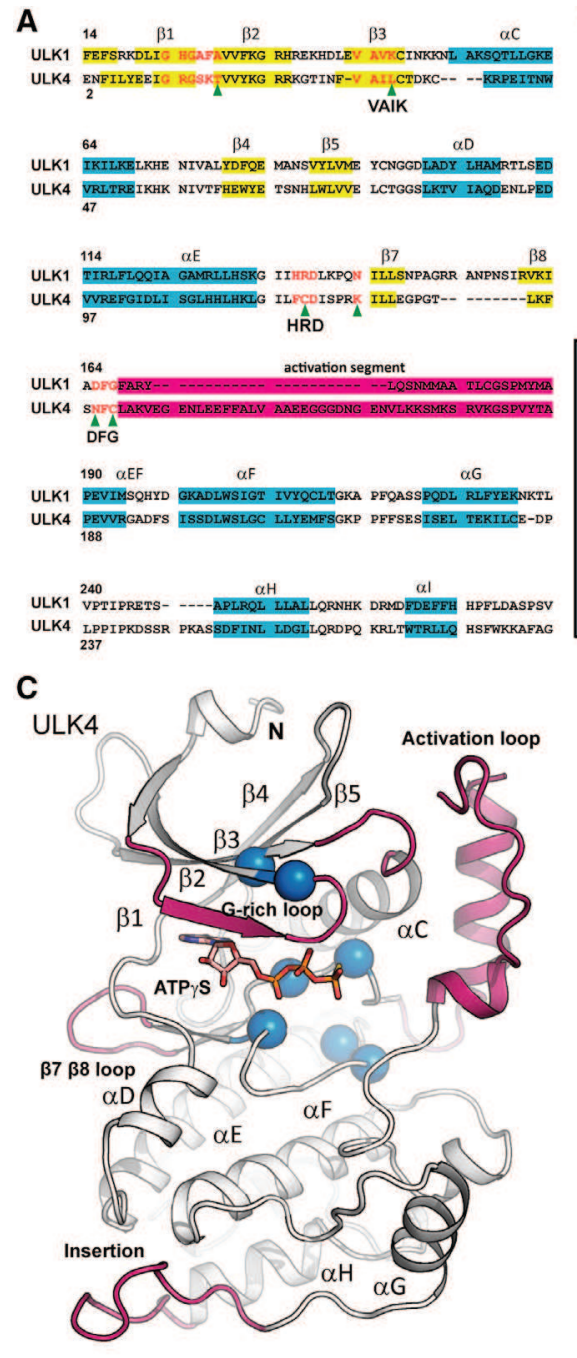

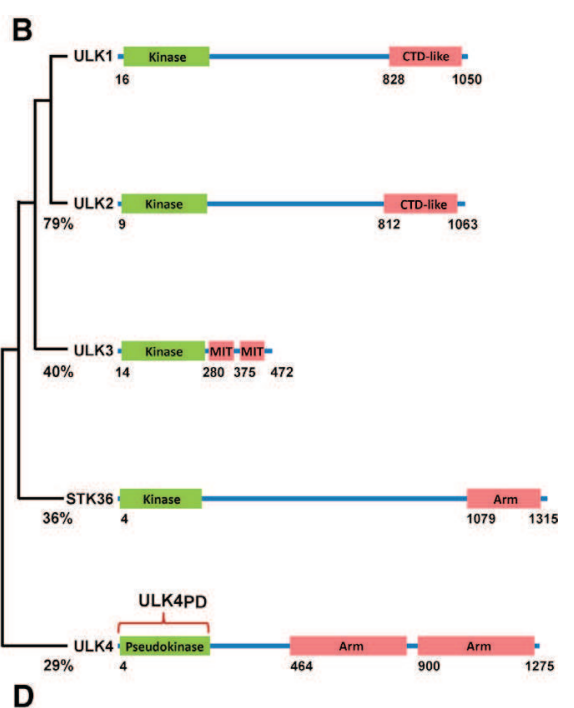

D

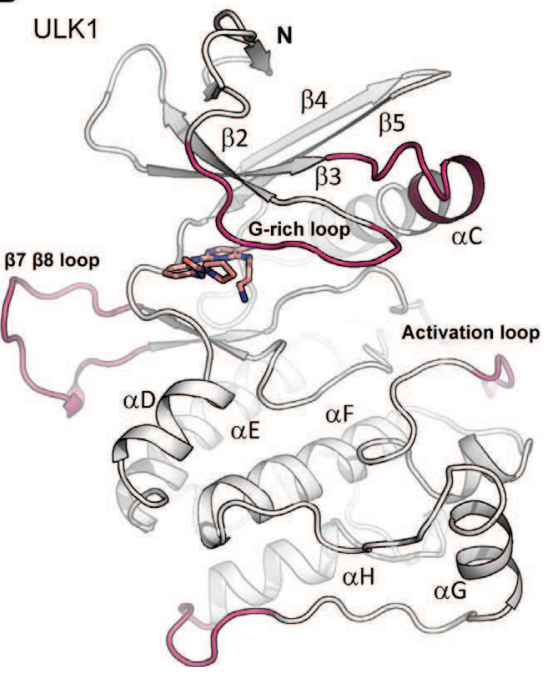

Figure 1. Structural Features of ULK Kinases

(A) Structure-based alignment of the kinase domains of ULK1 and ULK4. The main secondary structural elements are highlighted and conserved catalytic domain motifs are labeled. Amino acid residue changes in conserved motifs are indicated by a green arrow.

(B) Domain architecture of the human ULK family. Protein interaction domains are labeled as C-terminal domain (CTD) (ULK1 and ULK2), microtubule interacting and trafficking molecule (MIT) (ULK3), and armadillo repeat domain (Arm) (STK36 and ULK4).

(C) Structure of the ULK4 pseudokinase domain in complex with ATPrS. Positions of residues in conserved catalytic domain motifs that are altered in ULK4 are highlighted by blue spheres.

(D) Structure of ULK1 for comparison. Additional supplemental figures (Figure S2) show superimpositions of ULK4 with ULK1 and ULK2, respectively.

action partners. Because pseudokinases are often deregulated in diseases, a better understanding of pseudokinase interactions and their regulatory mechanisms would be desirable also for the evaluation of their potential as targets for the development of new therapeutics (Jacobsen and Murphy, 2017; Ribeiro et al., 2019).

Unc-51-like kinase 4 (ULK4) harbors sequence variations and lacks essential residues in all five conserved kinase motifs that are important for catalytic activity (Figure 1A). In particular, the substitutions of the VAIK lysine with leucine and the HRD histidine with phenylalanine are rare events in the human kinome. In addi-

kinase pathways through interactions with the surface of its pseudokinase C-lobe which constitutes a docking site for client proteins (Citri et al., 2003). When binding, the clients are forced into a specific conformation, resulting in client activation (Jura et al., 2009).

An intriguing example of a complex and dynamic regulatory mechanism mediated by a pseudokinase is mixed lineage kinase domain-like (MLKL), a key regulator of the non-apoptotic, kinase-dependent programmed cell death called necroptosis (Murphy et al., 2013). MLKL comprises an N-terminal four helix bundle (4HB) domain and a C-terminal pseudokinase, which lacks catalytic activity due to loss of the DFG and HRD motifs. Induction of necroptosis relies on two active kinases, the receptor-interacting protein kinases RIPK1 and RIPK3, which interact with MLKL forming the necrosome. Formation of this complex results in phosphorylation of MLKL, triggering a conformational change in MLKL that unleashes the $4 \mathrm{HB}$ domain resulting in membrane recruitment and formation of membrane-disrupting pores (Sun et al., 2012; Wang et al., 2014).

Unfortunately, for many pseudokinases there are no data available on the pathways they regulate and their specific inter- tion, the lack of the essential ATP binding motifs, such as VAIK (VAIL in ULK4) and DFG (NFC in ULK4) suggests that ULK4 has compromised ATP binding. However, strong ATP binding has recently been reported by a comparative binding study using temperature shift assays (Lucet and Murphy, 2017). A recent structure of the catalytic domain in complex with weakly binding inhibitors revealed a canonical kinase fold and may provide the foundation for the development of ULK4 inhibitors that modulate ULK4 scaffolding function (Khamrui et al., 2020).

The ULK family of kinases comprises the catalytically active members ULK1, ULK2, ULK3, and STK36. The kinase domains in ULKs are located at the $\mathrm{N}$ termini of all family members. Usually, the regions $\mathrm{C}$-terminal to the kinase domain contain protein interaction motifs important for substrate recruitment (Figure 1B). The role of the $\mathrm{C}$-terminal substrate recruitment domains has been demonstrated for ULK3 (Caballe et al., 2015). Its second microtubule interacting and trafficking domain binds to the protein IST1, thus enabling the ULK3 kinase domain to phosphorylate IST1 (Caballe et al., 2015). This mechanism enables ULK3 to regulate the timing of membrane remodeling by the ESCRTIII machinery. Analogously, the ULK1 C-terminal domain-like 
(CTD) domain interacts with autophagy-related protein 13 (ATG13). When activated, the ULK1 kinase domain phosphorylates the bound ATG13, which is part of the cascade ultimately leading to autophagosome formation (Zachari and Ganley, 2017). Despite its lack of kinase activity, a similar recruitment mechanism for substrates or interacting proteins might be also relevant for ULK4 which contains armadillo repeat protein interaction domains. However, no interaction partners for the ULK4 C-terminal armadillo repeats which are also present in C terminus of STK36 have been thus far reported.

ULK4 has been linked to several human disorders, highlighting the developmental function of this catalytically inactive kinase scaffold protein. Single-nucleotide polymorphisms (SNPs) in the ULK4 gene have been linked to blood pressure regulation and hypertension (Levy et al., 2009). As a consequence, certain ULK4 alleles increase the risk of acute aortic dissections (Guo et al., 2016). Genome-wide association studies (GWAS) have identified a ULK4 polymorphism associated with increased risk for the development of multiple myeloma (Broderick et al., 2011). Deletion of ULK4 in mice results in hydrocephalus, a condition linked to impaired cilia development (Vogel et al., 2012). Multiple roles for ULK4 in brain development and neuronal function have been reported, including neuronal motility (Lang et al., 2014), cortex development (Lang et al., 2016), hypomyelination (Liu et al., 2018b), and GABAergic signaling (Liu et al., 2018a). However, a molecular mechanism of how ULK4 mediates these cellular signaling functions is lacking. ULK4 falls within a class of "dark" kinases for which there is little or no functional data (Oprea, 2019).

To elucidate the mechanistic basis for ULK4 function, we solved the crystal structure of the ULK4 pseudokinase domain in complex with ATP $\gamma \mathrm{S}$ to gain insights into its unique nucleotide binding properties and identified interaction partners using mass spectrometry. The structure of the ULK4 pseudokinase domain revealed a unique nucleotide binding mode that results in tight metal ion independent binding of the co-factor, also providing a rationale for the disease-relevant polymorphisms affecting ATP binding residues. Through deep evolutionary analysis, we demonstrate that loss of catalytic activity of mammalian ULK4 occurred progressively in evolution with ancestral ULK4 orthologs in plants and protists retaining the canonical active site residues. Remarkably, the selective loss of ULK4 active site residues in mammals and metazoans correlated with the emergence of an extended activation segment that structurally compensates for the loss of the canonical salt bridge between the VAIK lysine and the $\alpha \mathrm{C}$ glutamate by stabilizing the regulatory $C$ helix in an active conformation. Identification of ULK4 binding partners by BiolD mass spectrometry suggested roles in microtubule and centrosome function. We propose specific docking/ protein-protein interaction sites by delineating the selective constraints imposed on the ULK4 catalytic domain.

\section{RESULTS AND DISCUSSION}

Based on secondary structure predictions and sequence alignments with ULK kinases we designed several constructs for expression in E. coli. Good yields of soluble protein were observed for the construct containing the pseudokinase domain residues 1-288 (ULK4 $\left.4_{\mathrm{PD}}\right)$. This sequence was expressed in frame with a cleavable (tobacco etch virus protease [TEV]) N-terminal purification tag $\left(\mathrm{His}_{6}-\mathrm{TEV}\right)$ allowing efficient purification of the recombinant protein. However, removal of the $\mathrm{N}$-terminal tag by TEV protease was difficult, indicating that the cleavage site was not accessible. The tag was therefore not removed for crystallization and functional studies. In size-exclusion chromatography, ULK4 $4_{\mathrm{PD}}$ eluted as a monomeric protein (Figure S1). The identity of ULK4 $4_{P D}$ was confirmed by mass spectrometry, confirming the correct mass of the un-cleaved recombinant protein, which however underwent $\mathrm{N}$-terminal methionine excision. No post-translational modifications were detected. The recombinant protein that was more than $95 \%$ pure as judged by SDSPAGE readily crystallized in the presence of non-hydrolyzable ATP $\gamma S$ yielding crystals that diffracted to $1.9 \AA$ resolution. Data collection statistics and refinement are summarized in Table 1.

Structural Features of the ULK4 Pseudokinase Domain

The structure of ULK $4_{\mathrm{PD}}$ revealed the canonical bilobal domain architecture of protein kinases (PDB: 6TSZ; Figure 1C) that was similar to a recent ULK4 structure in complex with an inhibitor (PDB: 6U5L; Khamrui et al., 2020), but a larger portion of the activation segment was visible in the ATP complex. The structures of the active ULK family members ULK1 and ULK2 superimposed well with ULK4 $4_{P D}$ despite the low sequence identity (29\%) shared between these two kinases (Chaikuad et al., 2019; Lazarus et al., 2015) (Figures 1D and S2). However, major structural differences were observed in the length of the $\alpha \mathrm{C}$ helix, the $\beta 7-\beta 8$ loop length, the length and topology of the activation loop, as well as the orientation of the $\alpha \mathrm{G}$ helix, which is also notably different (Figure 1). This helix does not form extensive crystal contacts in ULK structures and the ULK $4 \alpha G$ helix remained stable in a molecular dynamics (MD) study (see below), suggesting that the captured conformations in the compared crystal structures are also present in solution. The glycine-rich loop contains a threonine residue in ULK4 (T16) at a position wherein active kinases usually a glycine is found. The introduction of this bulky residue resulted in a rotation of the $\beta 1$ strand. A similarly rotated $\beta 1$ strand in combination with a glycine-rich loop threonine has been observed in structures of the TYK2, JAK1, and JAK2 pseudokinase domains (Lupardus et al., 2014). The canonical VAIK motif is replaced by VAIL in ULK4, replacing the conserved lysine residue by a leucine (L33). This substitution has not been reported for any other human kinase. In active kinase conformations, the VAIK lysine stabilizes the "in" conformation of helix $\alpha \mathrm{C}$ by forming a highly conserved salt bridge with an invariant glutamate present in $\alpha \mathrm{C}$. In addition, this lysine residue is essential for coordinating the nucleotide phosphates in the ATP bound state. Structural superimposition with ULK1 showed that the $\alpha \mathrm{C}$ glutamate residue is also missing in ULK4 and it has been replaced by a tryptophan (W46) (Figure 2A). The VAIL leucine is, however, too far away (4 $\AA$ ) to form efficient hydrophobic interactions with W46. Instead, W46 extends the regulatory spine through interaction with $\mathrm{F} 140$, the central residue in the degenerated DFG motif (NFC in ULK4), most likely contributing to the stability of the observed activelike state of the pseudokinase domain. It is interesting to note that the pseudokinase domain of STK40 is the only other human kinase that also bears an NFC motif (Durzynska et al., 2017). Nevertheless, $\alpha \mathrm{C}$ is in an active "in" conformation similar to 


\begin{tabular}{|c|c|}
\hline & ULK4-ATP $\gamma S$ \\
\hline \multicolumn{2}{|l|}{ Data Collection } \\
\hline Space group & P 1211 \\
\hline \multicolumn{2}{|l|}{ Cell dimensions } \\
\hline$a, b, c(\AA)$ & $62.44,41.47,69.76$ \\
\hline$\alpha, \beta, \gamma\left({ }^{\circ}\right)$ & $90.00,113.06,90.00$ \\
\hline Molecules/AU & 1 \\
\hline Resolution $(\AA)^{\mathrm{a}}$ & $36.3-1.90(1.97-1.90)$ \\
\hline Unique reflections ${ }^{a}$ & $25,013(2,518)$ \\
\hline Completeness (\%) & $95.2(96.4)$ \\
\hline Multiplicity ${ }^{a}$ & $3.4(3.3)$ \\
\hline $\mathrm{R}_{\text {merge }}(\%)^{\mathrm{a}}$ & $6.3(45.9)$ \\
\hline $\operatorname{CC}(1 / 2)^{a}$ & $0.997(0.862)$ \\
\hline Mean $I / \sigma(I)^{a}$ & $12.7(2.1)$ \\
\hline \multicolumn{2}{|l|}{ Refinement } \\
\hline $\mathrm{R}_{\text {work }},(\%)^{\mathrm{b}}$ & 20.1 \\
\hline$R_{\text {free }},(\%)^{b}$ & 25.3 \\
\hline \multicolumn{2}{|l|}{ No. of atoms } \\
\hline Protein & 2,232 \\
\hline Water & 83 \\
\hline Ligands/ions & 31 \\
\hline \multicolumn{2}{|c|}{ Root-mean-square deviation } \\
\hline Bonds $(\AA ̊)$ & 0.095 \\
\hline Angles $\left({ }^{\circ}\right)$ & 1.93 \\
\hline Mean $B\left(\AA^{2}\right)$ & 34.5 \\
\hline PDB & $6 \mathrm{TSZ}$ \\
\hline \multicolumn{2}{|c|}{$\begin{array}{l}\text { Values in parentheses are for the highest-resolution shell. } \\
{ }^{\mathrm{b}} \mathrm{R}_{\text {work }} \text { and } \mathrm{R}_{\text {free }}=\sum \| \mathrm{F}_{\text {obs }}|-| \mathrm{F}_{\text {calc }}|| \sum\left|\mathrm{F}_{\text {obs }}\right| \text {, where } \mathrm{R}_{\text {free }} \text { was calculate } \\
\text { with } 5 \% \text { of the reflections chosen at random and not used in th } \\
\text { refinement. }\end{array}$} \\
\hline
\end{tabular}

the one observed in phosphorylated ULK1 (PDB: 4WNO). Helix $\alpha \mathrm{C}$ is one turn shorter than in ULK1. However, the length in $\alpha \mathrm{C}$ is highly variable in kinases and may depend on the activation state (Eswaran et al., 2007). In ULK2, $\alpha$ C is even shorter than in ULK4 and contains a helical insert in the linker connecting $\alpha \mathrm{C}$ with $\beta 3$ (Figure S2). The $C$ terminus of $\alpha \mathrm{C}$ is stabilized by interactions of the degenerated NFC motif residue F140 which assumes an NFC "in" conformation.

The catalytic loop that comprises the conserved HRD motif in canonical kinases is replaced by an FCD motif in ULK4. However, the phenylalanine (F119) within the FCD motif still anchors the catalytic loop to the kinase core through hydrophobic interactions with residues L109, L112, I122, F137, and F140. The HRD arginine residue that often links the catalytic loop to phosphorylated residues in the activation segment is replaced by a cysteine, but this position is quite variable also in active kinases. The kinase catalytic aspartate, however, is conserved in ULK4. There is no indication that this aspartate can function as a catalytic base, but it still forms a commonly seen salt bridge to the +5 residue (K126) in the catalytic loop. This residue in the very $\mathrm{C}$ terminus of the catalytic loop is typically an asparagine in canonical kinases, but it is a lysine (K126) in ULK4. As a result, there is a network of salt bridges linking K126 with the co-factor ATP and D121 in the catalytic loop. To date, the only other pseudokinase for which an aspartate to lysine salt bridge in the catalytic loop has been reported is TRIB1 which maintains a similar catalytic loop conformation as observed in ULK4 (PDB: 5CEM). The salt bridge is, however, tilted toward the core of TRIB1 due to a side chain flip of TRIB1 D205 (Murphy et al., 2015).

In comparison with the canonical kinase fold, the ULK4 activation segment contains a large insert with a helical structure (L150-E161). The activation segment $\mathrm{N}$ terminus contains the unusual degenerated DFG motif (NFC) followed by a typical short sheet region that anchors that activation segment to the lower kinase lobe by hydrogen bonds formed by main-chain interactions between two antiparallel short $\beta$ sheets. At the $N$ terminus of the short sheet structure there is a large helix inserted that forms an intricate network of salt bridges with $\alpha \mathrm{C}$ possibly stabilizing its inward oriented conformation. The interaction with $\alpha \mathrm{C}$ is further stabilized by hydrophobic interactions that form a small hydrophobic core involving residues F153, F154, and L150 at the center of this activation segment helix and P41, L49 located in $\alpha \mathrm{C}$. A salt bridge links E148 located in the loop N-terminal to the activation segment helix with $\alpha \mathrm{C}$ R48. The hydrophobic environment surrounding this salt bridge suggests a strong polar interaction of the R48/E148 salt bridge (Figure 2B). The helix is connected to a coil structure bypassing the catalytic loop. Intriguingly, S182 located in the activation segment, five residues $\mathrm{N}$-terminal to the APE helix, is positioned opposite the catalytic base of the degenerated HRD motif (FCD) mimicking a substrate bound state. Thus, the substrate binding site in ULK4 is blocked by the activation segment. The activation segment terminates with a canonical APE motif.

\section{Degradation of the Active Site has Co-evolved with an Extended Activation Segment}

We performed phylogenetic analysis of ULK4 orthologs from diverse organisms to investigate the origin and evolution of ULK4 as a pseudokinase. We identified ULK4 orthologs from diverse taxonomic groups, including metazoans, plants, and protists. Phylogenetic analysis of these sequences reveals clear separation of metazoan ULK4 from plants and protists (Figure S3). Further annotation of the sequences based on the presence or absence of the canonical active residues indicates species-specific variations in the canonical active site residues, namely K72, E91, D166, and D184 (protein kinase A numbering). While metazoan ULK4s lack three of these canonical residues except for the catalytic aspartate (D166) (cyan circular stripes in Figure S3), orthologs in plants and protists conserve these canonical residues with few exceptions, suggesting that loss of catalytic function of ULK4 emerged later in evolution, presumably for developing specialized metazoan ULK4 pseudokinase functions. In addition to divergence in the canonical active site residues, metazoan ULK4s displayed variations in the activation loop as well. Comparison of the activation loop lengths across ULK4 orthologs indicates an extended activation segment (mean length of 49.6 amino acids) in pseudo metazoans compared with ULK4 orthologs from other species (Figures 2C, S3, and S4).

The extended segment (L150-E161) forms a helical insert in human ULK4 and packs against the $\mathrm{C}$ helix in the crystal structure. To test the stability of this conformation, we performed 
A

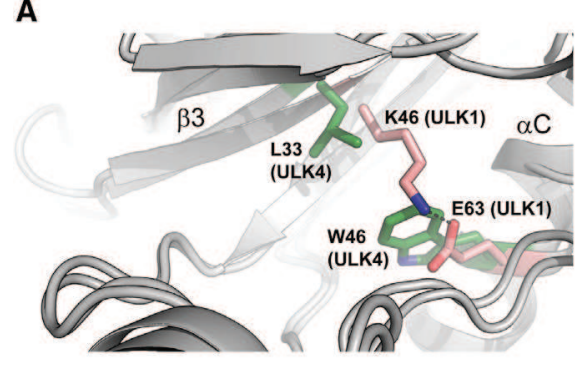

C

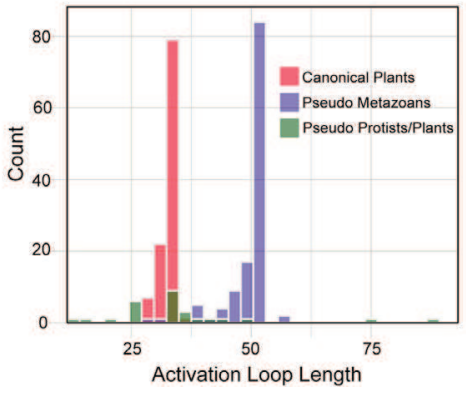

B

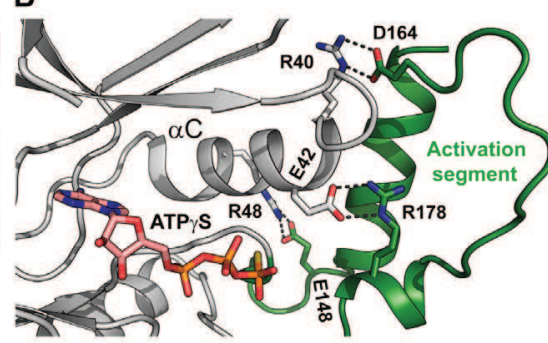

D $\overrightarrow{\mathrm{\omega}}$

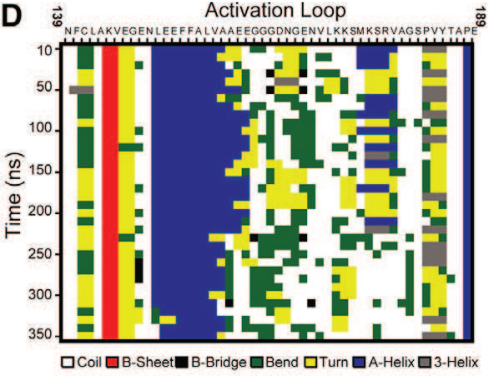

Figure 2. Details of ULK4 Structural Features and Evolutionary Conservation

(A) Comparison of interaction between the ULK1 VAIK motif and $\alpha \mathrm{C}$ showing the canonical salt bridge of $\alpha \mathrm{C}$ in conformations and ULK4 in which the VAIK lysine is replaced by a leucine residue (L33) and the $\alpha \mathrm{C}$ glutamate by a tryptophan (W46). (B) Details of the interaction of the ULK4 activation segment helix with $\alpha \mathrm{C}$. Interactions in the catalytic loop are also shown.

(C) Histogram showing the lengths of the activation loop in ULK4 orthologs. Canonical plants, pseudo metazoans, and pseudo protists/plants are colored as light red, light purple, and green, respectively.

(D) Secondary structure plot of the activation loop of ULK4. Define secondary structure of proteins (DSSP) was used to define the secondary structure. A maximum likelihood tree of available ULK4 sequences of different species is shown in Figure S3.
MD simulation of human ULK4 in the presence of ATP (PDB: 6TSZ). The simulation indicates that, while the inserted helical segment (L150-V157) stably packs against the $\mathrm{C}$ helix, the rest of the loop (A158-E161) is flexible, as shown by the lack of secondary structure propensity during the course of simulation (Figure 2D). A helical activation loop has also been reported for mouse MLKL (PDB: 4BTF) (Murphy et al., 2013). This helical segment assumes, however, a different orientation compared with the ULK4 activation helix. In the mouse MLKL structure, the $\alpha \mathrm{C}$ helix is moved outward making room for the MLKL activation segment helix, which resides in a similar position as the ULK4 $\alpha \mathrm{C}$ helix. It is interesting to note that, in human MLKL, no helical insert has been reported. These data suggest that $\alpha \mathrm{C}$ and the activation loop helix may serve pseudokinase-specific functions that may even differ between species, as observed for MLKL (Petrie et al., 2018). Our evolutionary analysis suggests that the loss of the lysine-glutamate salt bridge in metazoan ULK4 required the stabilization of $\alpha \mathrm{C}$ by the activation segment helix, which in metazoan ULK4 has co-occurred with an extended activation loop, presumably compensating for the loss of the canonical K-E salt bridge. However, since the motif "LEEFFALVAAEE" forming the helical insert in human ULK4 is present in metazoan ULK4 pseudokinases, it is likely that the helical activation loop structure is a common feature of ULK4 sequences that lack the $\beta 3$-lysine.

\section{Evolutionary Constraints Distinguishing ULK4 from Other Paralogs}

We next performed a Bayesian statistical analysis to identify the evolutionary constraints distinguishing ULK4 from the closely related ULK1-3 and STK36 sequences. This revealed strong ULK4-specific constraints imposed on residues in the $\mathrm{N}$-terminal ATP binding lobe of the pseudo kinase domain. Specifically, these constraints map to residues in the $\beta 2-\beta 3$ loop as well as in $\beta 4$ and $\beta 5$ strands. Some of these constrained residues include R22, R23, and K24, which form a sol- vent-exposed surface patch with hydrophobic residues $\mathrm{I} 27$ and F29, which are also ULK4 specific (Figure 3A). In addition, an extended network of ULK4-specific residues (F61, W64, E66, and L71) structurally couple the surface patch to the $C$ helix. These residues are conserved across diverse ULK4 orthologs (including plants and protists) and divergent from the closely related ULK1-3 and STK36 (Figure 3B), implying that they are constrained for important ULK4-specific functions. A possible role of this ULK4-specific surface patch could be that it serves as a docking site similar to the one reported for Aurora A with the microtubule-associated protein TACC3 (Burgess et al., 2018).

\section{ULK4 Has an Unusual ATP Binding Mode}

Because conserved motifs known to be essential for nucleotide binding are absent in ULK4, we were particularly interested in how ULK4 interacts with the co-factor ATP. After purification of the recombinant protein the absorbance spectra of ULK $4_{P D}$ showed an unusual ratio at 260 and $280 \mathrm{~nm}\left(\mathrm{~A}_{260} / \mathrm{A}_{280}\right)$ suggesting co-purification of nucleotides. Most proteins exhibit maxima at $280 \mathrm{~nm}$ due to the presence of tryptophan residues, with the $A_{260} / A_{280}$ ratio usually ranging from 0.45 to 0.55 . In ULK4 $4_{P D}$, this ratio was 0.76 (Figure $4 \mathrm{~A}$ ). An explanation for an aberrant $\mathrm{A}_{260} / \mathrm{A}_{280}$ ratio might be the amino acid composition of the protein. This is not the case in ULK4 $4_{\mathrm{PD}}$, which contains $2.0 \%$ tryptophan residues. Another explanation might be the covalent attachment of a co-factor, such as ADP-ribose, but this scenario can be excluded because any covalent modification would have been detected in our mass spectrometry analysis. We therefore assume that ULK4 $4_{\mathrm{PD}}$ tightly, but non-covalently, bound to a nucleotide metabolite from $E$. coli, and that this interaction was still present at least partially after dialysis and several chromatographic purification steps.

To assess the durability of ATP binding we performed a MD simulation of the crystal structure of ULK4 with ATP bound to it. The co-factor remained stably bound during this 350-ns 


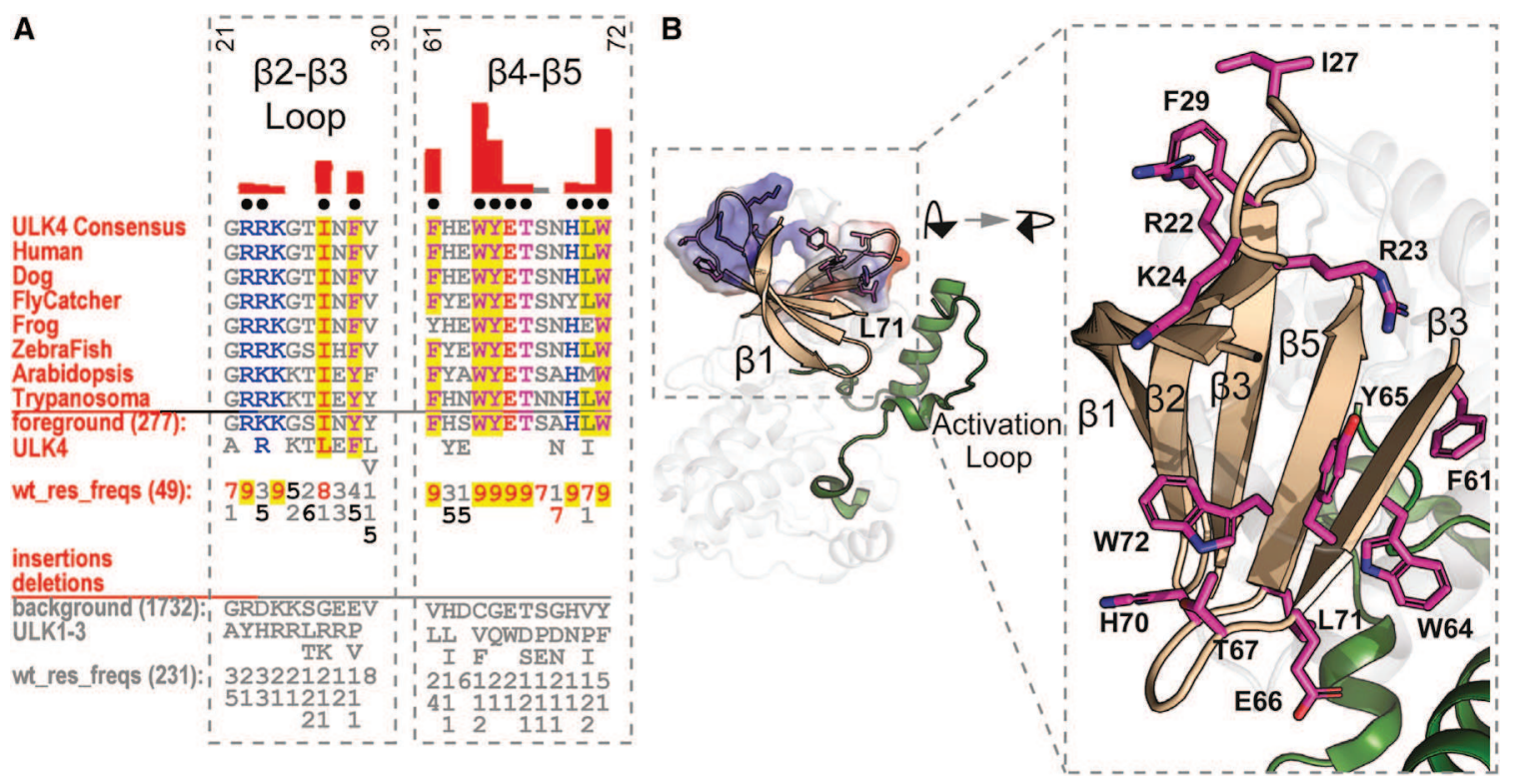

Figure 3. ULK4 Specific Constraints Are in the N-Lobe

(A) Sequence constraints that distinguish ULK4 sequences from closely related ULK1-3 sequences are shown in a contrast hierarchical alignment (CHA). The CHA alignment shows selected ULK4 sequences from diverse organisms as the display alignment. The foreground alignment consists of 277 ULK4 sequences while the background alignment contains 1,732 ULK1-3 sequences. The foreground and background alignments are shown as residue frequencies below the display alignment in integer tenths (1-9). The histogram (red) above the display alignment indicates the extent to which distinguishing residues in the foreground alignment diverge from the corresponding position in the background alignment. Black dots mark the alignment positions used by the BPPS (Neuwald, 2014) procedure when classifying ULK4 from other ULK sequences. Alignment number is based on the human ULK4 sequence.

(B) ULK4-specific residues mapped onto the crystal structure of human ULK4. The distinguishing residues are shown as sticks and surface electrostatic. The activation loop is colored green. A detailed view is shown in the right panel. Carbon atoms of the residues are colored in magenta. A consensus sequence of ULK4 activation segment is shown in Figure S4.

simulation supporting the high affinity of the co-factor (Figure S5). A video illustrating structural changes is also available in the Supplemental Information.

To identify nucleotides that are most likely bound to ULK4 in cells, we performed a co-factor screen using the most common nucleotide-based co-factors and their counter ions. For this analysis we performed thermal shift assays, a screening technology that is well established in our laboratory and that detects molecular interactions based on their effects on protein stability (Fedorov et al., 2012). Recombinant ULK4 $4_{\mathrm{PD}}$ exhibited a melting temperature $\left(T_{M}\right)$ of $30^{\circ} \mathrm{C}$. In comparison with most kinases $\left(40^{\circ} \mathrm{C}<\mathrm{T}_{\mathrm{M}}<60^{\circ} \mathrm{C}\right) \mathrm{ULK} 4_{\mathrm{PD}}$ was highly unstable after purification. Both adenine and guanine nucleotides strongly stabilized ULK4 $4_{P D}$. By far the highest $T_{M}$ shift was observed using ATP $\left(20^{\circ} \mathrm{C}\right)$, while the ADP stabilization was $9^{\circ} \mathrm{C}$. This highlighted the importance of the ATP $\gamma$-phosphate for binding. ADP derivatives, such as ADP-ribose and acetyl-CoA stabilized to a similar extent as ADP alone, indicating that the substituents did not interfere strongly with binding (Figure 4B). Interestingly, $\mathrm{Mg}^{2+}$ ions did not promote nucleotide binding in accordance with previously published results (Murphy et al., 2014). In contrast, the presence of $5 \mathrm{mM} \mathrm{Mg}^{2+}$ ions significantly destabilized the ULK $4_{\mathrm{PD}}-\mathrm{ATP}$ complex by $8^{\circ} \mathrm{C}$. The pseudokinases calcium/ calmodulin-dependent serine protein kinase, STE20-rlated adaptor beta (STRAD $\beta$ ), MLKL pseudokinase, and ephrin receptor B6 all lack a functional DFG motif and show metal-independent co-factor binding (Bailey et al., 2015; Mukherjee et al., 2008; Murphy et al., 2014). Since ATP is highly abundant in cells, it is likely that the physiological ligand of ULK4 $4_{\mathrm{PD}}$ is indeed ATP. We therefore used the highly stabilizing non-hydrolysable ATP analog ATP $\gamma S$ for crystallization studies. Screening of a kinase targeted library of 1,500 compounds using differential scanning fluorimetry (DSF) resulted only in small temperature shifts for some inhibitors (data not shown) suggesting weak interaction with typical kinase inhibitors.

An intriguing aspect of the ULK4 ${ }_{P D}-A T P \gamma S$ structure was the unusual binding mode of the nucleotide. In an active kinase, both the ATP orientation and the triphosphate moiety need to be adjusted perfectly to allow for catalysis. For instance, in the first structure of a kinase ATP complex, protein kinase A (PDB: 1ATP), the co-factor binds two $\mathrm{Mg}^{2+}$ ions coordinated by the ATP phosphates, the DFG aspartate (D184), the catalytic base in the HRD motif (D166), and N171 and K168 located in the catalytic loop. The glycine-rich loop interacts closely with the ATP phosphates forming several main-chain interactions. The adenine ring is anchored to the hinge region by two conserved hydrogen bonds with main-chain atoms of E121 and V123 (Figure 4C). While some interactions between ULK4 and ATP $\gamma$ S are inspired by the kinase ATP binding mode, other interactions have not been described in active kinases and pseudokinase co-factor complexes before (Figure 4D). Similar to active kinases, the ATP adenine is anchored to the ULK4 hinge main chain by hydrogen bonding to E76 and C78. Hydrophobic interactions stabilize the adenine ring system which is sandwiched between hydrophobic residues in the $\beta 2$ strand (V18), the VAIL motif (A31 and L33), as well as the $\beta 7$ strand 

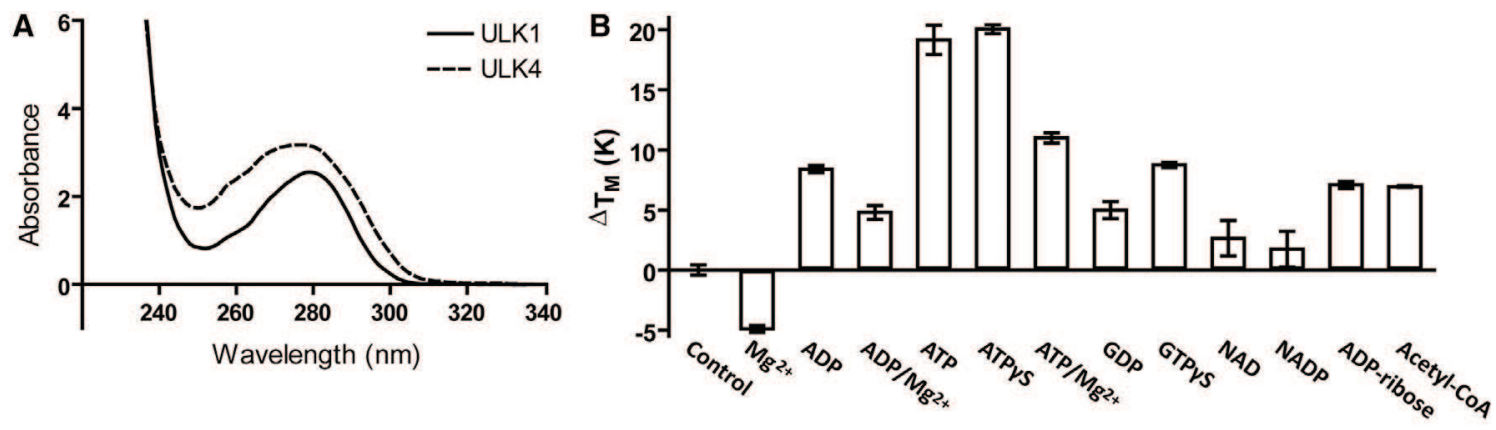

C

PKA

D

ULK4
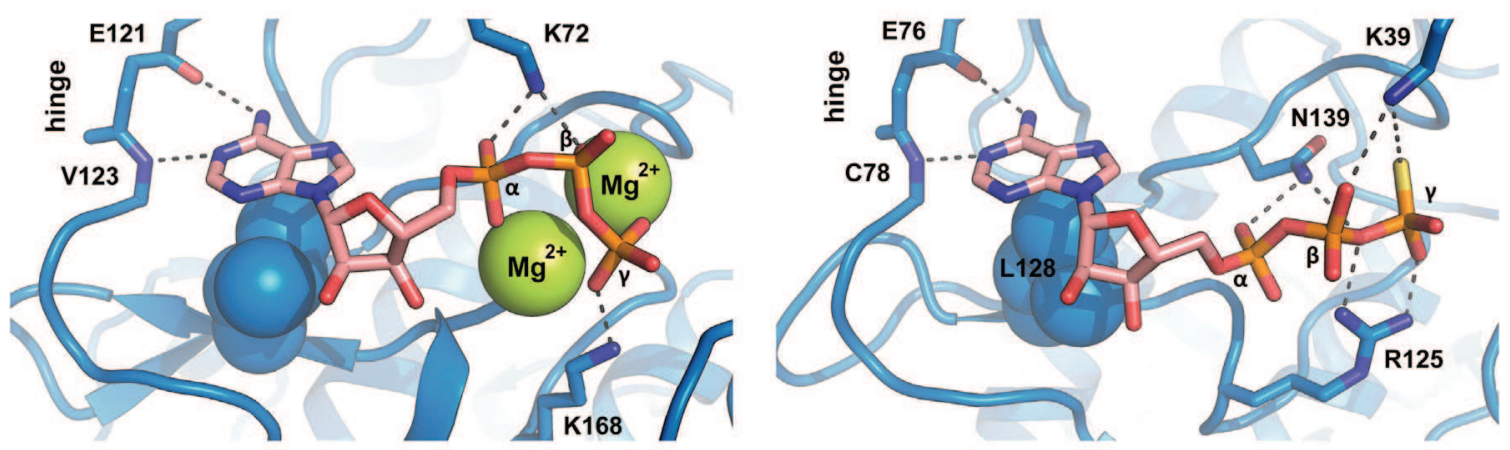

E

STRADa

$\mathbf{F}$
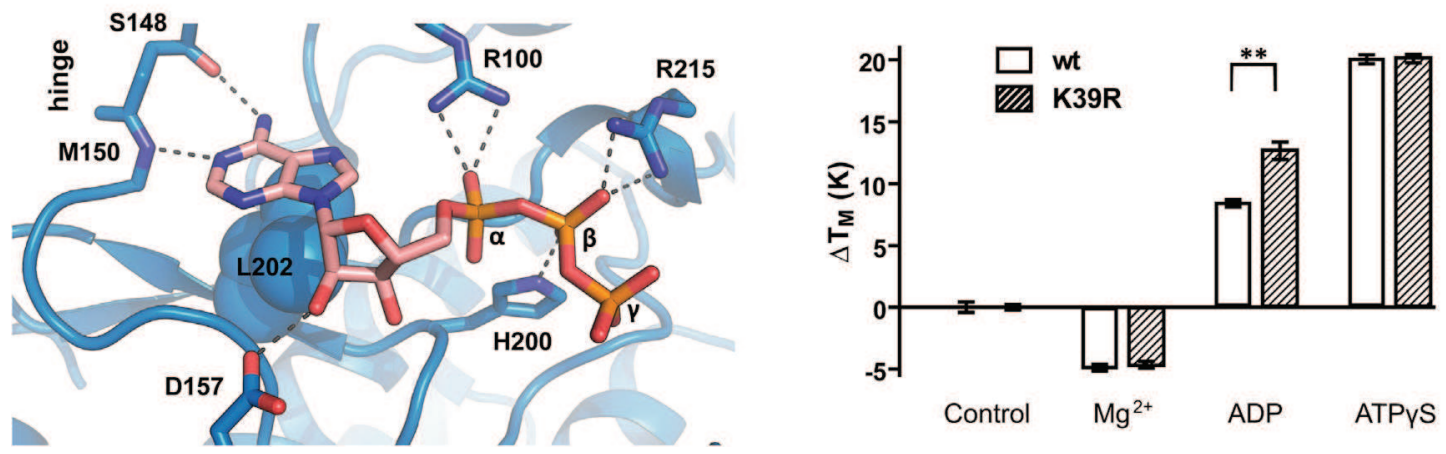

Figure 4. Nucleotide Binding to ULK4

(A) Comparison of UV spectra of ULK1 and ULK4. The high absorbance of ULK4 at $260 \mathrm{nM}$ suggests the presence of ATP after purification from bacteria.

(B) Affinity of nucleotides and effect of metals on binding. Shown are temperature shifts at $1 \mathrm{mM}$ nucleotide concentration as well as SEM $(n=3)$.

(C) Canonical binding mode of ATP exemplified by the protein kinase A (PKA) ATP complex (PDB: 1ATP). Main interactions are shown in stick representation and are labeled. Some side chains have been deleted for clarity. Major hydrophobic contacts interacting with the adenine ring in the low lobe are shown as spheres.

(D) Binding mode of ATP $\gamma$ S in ULK4. Orientations and labels are similar as shown in (C).

(E) Nucleotide binding in STRAD $\alpha$ (PDB: $3 G \mathrm{GNI})$.

(F) Effect of K39R polymorphism on nucleotide binding assess by temperature shift experiments. Shown are temperature shifts at $1 \mathrm{mM}$ nucleotide concentration as well as SEM $(n=3)$.

(L128). Intriguingly, the lack of the VAIK motif lysine is compensated by K39, capping the $\alpha \mathrm{C}$ helix. The ATP $\gamma \mathrm{S}$ triphosphate is embedded into an intricate hydrogen-bonding network involving side chains from the tip of $\alpha \mathrm{C}$ helix (K39), the catalytic loop (R125 and K126), and the DFG motif (N139). As a result, the ATP $\gamma$ S molecule decorates the back of a groove in ULK4 $4_{\mathrm{PD}}$ with the ribose hydroxyl groups and several phosphate oxygen atoms exposed. A similar groove is observed in the pseudokinase STRAD $\alpha$. Binding of both ATP and the allosteric activator protein MO25 allegedly forces STRAD $\alpha$ into an active-like conformation (Figure 4E). The ternary complex then binds to and activates the client kinase STK11/LKB1 (serine/threonine kinase 11/liver kinase B1) (Zeqiraj et al., 2009b). In this process, the role of ATP is solely to maintain STRAD $\alpha$ in its bindingcompetent form. The ATP molecule interacted directly neither with the client kinase nor with the activator.

Recently, GWAS linked several SNPs in ULK4 to diseases such as high blood pressure (Ehret et al., 2016; Levy et al., 2009) and sporadic thoracic aortic dissection (Guo et al., 2016). Intriguingly, SNP rs2272007 is localized in the 


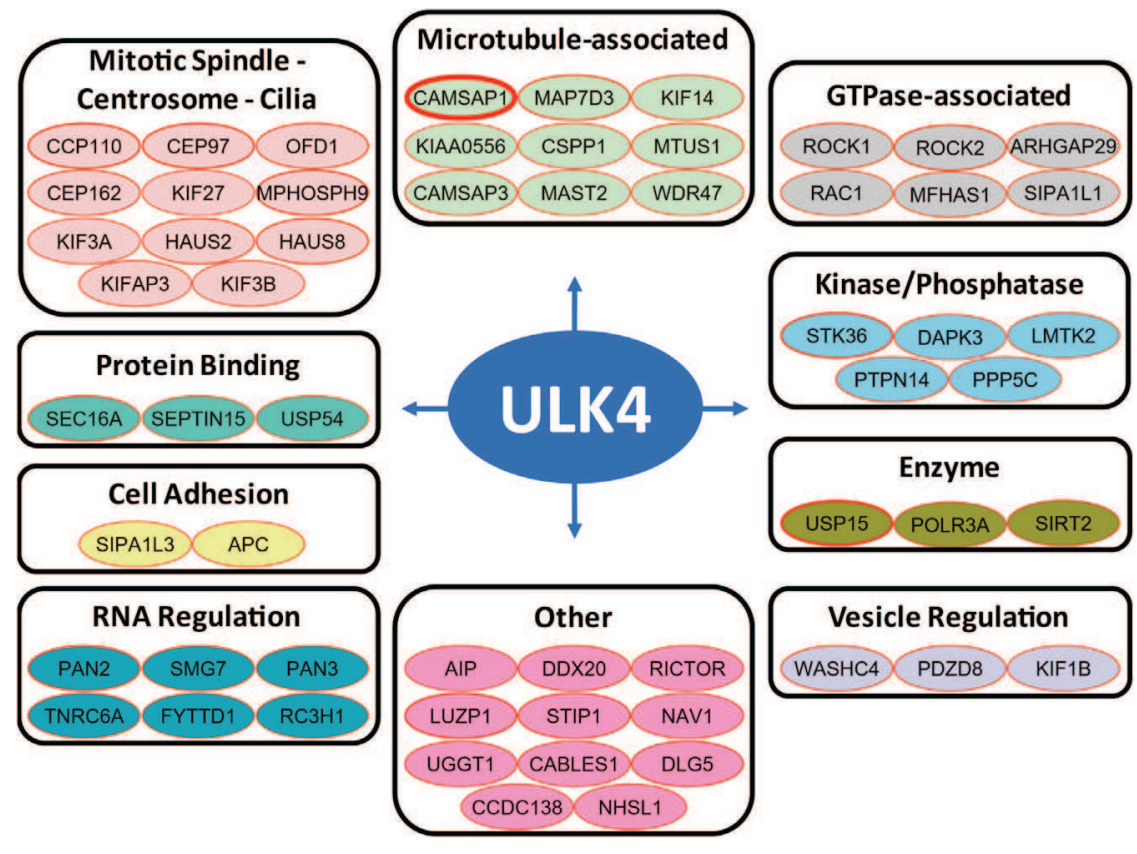

Figure 5. ULK4 Proximity Interaction Network

Proximity interactors of FlagBirA*-ULK4 and/or ULK4-BirA*Flag fusion proteins expressed in FlpIn T-REx 293 cells are shown. Probability of specific interactors was determined using Bayesian statistics against a panel of FlagBirA* only BiolD samples. Proteins with a Bayesian false discovery rate of interaction of $\leq 0.01$ were considered statistically significant putative proximity interactors. Border (in red) thickness of nodes is proportional to the number of peptide spectrum matches for each protein. Proteins are categorized according to gene ontology-based functions. ULK4 cellular localization data are shown in Figure S6. See also Table S1.

\section{BiolD Identifies Interaction \\ Partners of ULK4}

To the best of our knowledge, no validated ULK4 interacting partners have been reported. Knockdown studies revealed a role in neural cell migration pointing to a cytoskeletal function as

pseudokinase domain of ULK4 affecting residue 39. As described above, in the most common ULK4 variant, K39 takes over the role of the missing VAIK lysine. According to The 1000 Genomes Project, $68 \%$ of sequenced individuals carry the nucleotide T in rs2272007 and thus express ULK4 protein with a lysine in position 39 , and $32 \%$ carry the nucleotide C and express an arginine in this position. Therefore, we were interested in how the R39 polymorphism could affect ATP binding, and generated a site-directed mutant carrying this mutation. We also produced another reported variant: N139L. This variant protein was unstable during purification and precipitated quickly, precluding analysis. However, since N139 replaces the aspartate in the degenerated ULK4 DFG motif and the N139 side chains form intricate interactions with the ATP alpha and beta phosphates, the mutation into a hydrophobic lysine is likely to severely compromise ATP binding affecting the structural stability of the ULK4 pseudokinase domain. Temperature shift experiments using the $\mathrm{R} 39$ variant showed that the un-ligated protein has similar stability as the K39 protein, and the presence of $1 \mathrm{mM}$ ATP resulted in similar temperature shifts, suggesting that both lysine and arginine can form efficient salt bridges to the co-factor and that there was enough space in the ATP binding pocket to accommodate the slightly bulkier arginine side chain. Interestingly, a significant difference was observed in ADP binding. While ULK $4_{P D} \mathrm{~K} 39$ was stabilized by only $9^{\circ} \mathrm{C}$, ULK $4_{P D} R 39$ was stabilized by $13^{\circ} \mathrm{C}$, suggesting tighter binding of $A D P$. It is likely that the side chain of K39 is too short to form efficient salt bridges with the ADP $\beta$-phosphate, but R39 has sufficient length to form more efficient contacts (Figure 4F). Thus, ULK4 K39 and R39 differed in their affinities for ADP, whereas the variant N139L was highly unstable due to compromised ATP binding, highlighting the importance of ATP binding for ULK4 function and making a compelling case that altered nucleotide affinity is possibly responsible for disease development, as suggested by GWAS. well as a stimulating role on MAPK signaling (Lang et al., 2014). To better understand the ULK4 interaction landscape, we performed BiolD (proximity-dependent biotin identification) (Roux, 2013), a proximity-dependent labeling technique that identifies proteins in the vicinity of a target polypeptide in living cells. The technique makes use of a mutant form of the $E$. coli biotin ligase enzyme BirA* $(\mathrm{R} 118 \mathrm{G})$, which is fused to a protein of interest. The R118G BirA mutant is unable to maintain an interaction with the activated biotinoyl-AMP molecule. This highly reactive intermediate is thus released into the vicinity of the fused bait protein and reacts with amine groups of nearby polypeptides. Biotinylated proteins can then be purified using streptavidin, and identified by mass spectrometry (Gingras et al., 2019). N- and C-terminally FlagBirA*-tagged full-length ULK4 was expressed in Flp-In T-REx 293 cells. Immunofluorescence (anti-Flag) indicated that the tagged ULK4 protein is localized in the cytoplasm (Figure S6), in agreement with data available at the human protein atlas (https://www.proteinatlas.org).

ULK4 BiolD was performed using both $\mathrm{N}$ - and C-terminally tagged FlagBirA* fusion proteins, and each analysis was performed twice. Data were compared with BiolD conducted on 293 Flp-In T-REx 293 cells expressing the FlagBirA* tag alone to control for endogenously biotinylated proteins and polypeptides that interact non-specifically with the streptavidin-sepharose matrix material. Data were analyzed using the SAINT (statistical analysis of interactomes [Choi et al., 2011; Teo et al., 2014]) algorithm to identify high-confidence proximity interactors (displaying a Bayesian false discovery rate $\leq 0.01$ Figure 5 ; Table S1). The proximity interactor detected with the highest number of peptides was calmodulin-regulated spectrin-associated protein 1 (CAMSAP1), a large cytoplasmic scaffolding polypeptide that binds microtubules via its CKK domain, and controls noncentrosomal MT minus-end dynamics (Atherton et al., 2019; Baines et al., 2009). CAMSAP family members are involved in 
the regulation of a number of key cellular functions, including cell polarity, regulation of neuronal differentiation, and axonal regeneration (Akhmanova and Hoogenraad, 2015; Marcette et al., 2014; Pongrakhananon et al., 2018). The related family member CAMSAP3 was also identified as a ULK4 proximity interactor. Intriguingly, many tubulin binding and centrosomal proteins were also detected, including: HAUS2 and HAUS8 (HEC1/ NDC80-interacting centrosome-associated protein), components of the microtubule-binding augmin/HAUS complex, which is essential for mitotic spindle assembly (Goshima et al., 2008; Uehara et al., 2009); centrosomal proteins, such as CCP110 (centriolar coiled-coil protein 110), CEP97 (centrosomal protein 97), CSPP1 (centrosome and spindle pole-associated protein 1), and OFD1 (OFD1 centriole and centriolar satellite protein); and motor proteins of the kinesin family (KIF1B, KIF3B, and KIFAP3).

Other interacting partners included several protein kinases implicated in microtubular function, such as the microtubuleassociated serine/threonine kinase 2, Rho-associated coiledcoil-containing protein kinase 2 (ROCK2), and Rho GTPase-activating protein 29 (ARHGAP29). In addition, PTPN14 (protein tyrosine phosphatase, non-receptor type 14) and the ULK family member STK36, an important regulator of the sonic hedgehog pathway (Han et al., 2019; Murone et al., 2000), were also detected as high confidence proximity interactors.

Consistent with the BiolD data, CAMSAP1, PTPN14, ROCK1, and ROCK2 co-migrated with full-length C-terminal Flag-tagged ULK4 in analytical ultra-centrifugation density gradients, as detected by western blotting (Figure 6A). Co-immunoprecipitation using two different methods (C-terminal FLAG tag IP and streptavidin pull-down) validated the ULK4 interactions with CAMSAP1, ROCK1, ROCK2, and PTPN14 (Figures 6B and 6C). The same interactions were validated using $\mathrm{N}$-terminal FLAG tag constructs with similar results (Figure S7).

To map the regions of ULK4 that mediate these interactions, the pseudokinase domain and the armadillo repeat region were separately subjected to BiolD (Figure 6D). This analysis revealed that the armadillo repeat region interacted uniquely with CAMSAP1, OFD1, poly(A)-specific ribonuclease subunit 2 (PAN2), and several other proteins. ULK4 pseudokinase domain-specific interactions were detected for STK36, PTPN14, and CAMSAP3. The interaction of ULK4 pseudokinase domain with STK36 is particularly intriguing as, similar to STRAD/LKB1, this interaction might indicate that ULK4 directly regulates an active kinase (Zeqiraj et al., 2009a). All domain-specific detected interactions are summarized in Table S2. To determine whether the polymorphism at position 39 affects the ULK4 protein interaction network that might explain the pathogenicity of this amino acid alteration, the R39 variant was also subjected to BiolD. No significant changes in the interactome were detected (Table S3). Based on our DSF binding study, the R39 variant also bound ATP with similar affinity to the K39 protein. Stability of the pseudokinase domain fold is thus unlikely to be affected by this amino acid change.

The presented structural, bioinformatics, biochemical data together with our BiolD interaction study provided a diversity of high-confidence domain-specific interactions and suggested a microtubular and centrosomal function of ULK4. The high-affinity interaction with ATP is required for the stability of the pseu- dokinase domain, maintaining these interactions. The high-resolution structure of the ULK4 ATP $\gamma \mathrm{S}$ complex revealed a highly unusual ATP binding mode despite the lack of canonical ATP interaction motifs, demonstrating how this unusual pseudokinase redesigned interaction for co-factor binding. Our evolutionary studies indicated that ULK4 orthologs in plants and protists conserve the canonical active site residues, suggesting that they function as "active" kinases. In contrast, the metazoanspecific variations in the active site suggest that loss of kinase activity and emergence of ULK4 as a pseudokinase occurred later in evolution, presumably to accommodate the specialized functions of differentiated cell types, such as the metazoan-specific nervous and immune systems. We hope that the presented data will stimulate future research on the function of this poorly studied pseudokinase.

\section{SIGNIFICANCE}

ULK4 is an understudied protein associated with diseases such as hypertension and psychiatric disorders. Even though the overall structure of ULK4 has been reported previously (Khamrui et al., 2020), we provided in this study important data comprising structural details of the ULK4 ATP interaction in the absence of conserved canonical ATP binding motifs, evolutionary aspects, as well as interaction partners of ULK4. Despite alterations in ATP binding motifs that are essential in canonical kinases, many pseudokinases bind ATP or mimic an ATP bound state, which may not require or is even opposed by $\mathrm{Mg}^{2+}$ binding (Murphy et al., 2014) (Scheeff et al., 2009). In ULK4, polymorphism of key ATP binding residues, such as K39R and N139L, have been linked to disease development, suggesting that ATP binding is required for ULK4 function. Interaction of the ATP co-factor in canonical kinases leads to a closed structure that is considerably more stable. Therefore, it is likely that ATP binding, and the significant stability increase, is required for ULK4 scaffolding function and its role in cellular signaling. Additional structural elements co-emerged with the loss of canonical motifs in evolution that compensate for the loss of stabilizing effects. For instance, our interspecies analysis of ULK4 sequences showed that alteration of the active site motifs in metazoan ULK4 has evolved with an extended activation loop, which stabilizes the flexible $\mathrm{C}$ helix and compensates for the loss of VAIK lysine and the $\alpha \mathrm{C}$ glutamate. The diversity of structural mechanisms that show how pseudokinases maintain strong binding activity for ATP in the absence of canonical binding motifs is fascinating and suggest an essential role of stable domain structures in pseudokinases, which we confirmed by MD simulations. To shed light on signaling pathways regulated by ULK4 we performed interaction studies using BiolD mass spectroscopy. These data together with biochemical validation revealed high confidence interaction partners that were mapped to the pseudokinase and to the armadillo repeat domains, respectively. Many of the identified ULK4 interaction partners have centrosomal function, suggesting a centrosomal role of ULK4. In addition, a number of active kinases have been identified as ULK4 interactors, including the ULK family member STK36. Our studies provide a structural framework for targeting ULK4 in diseases. 


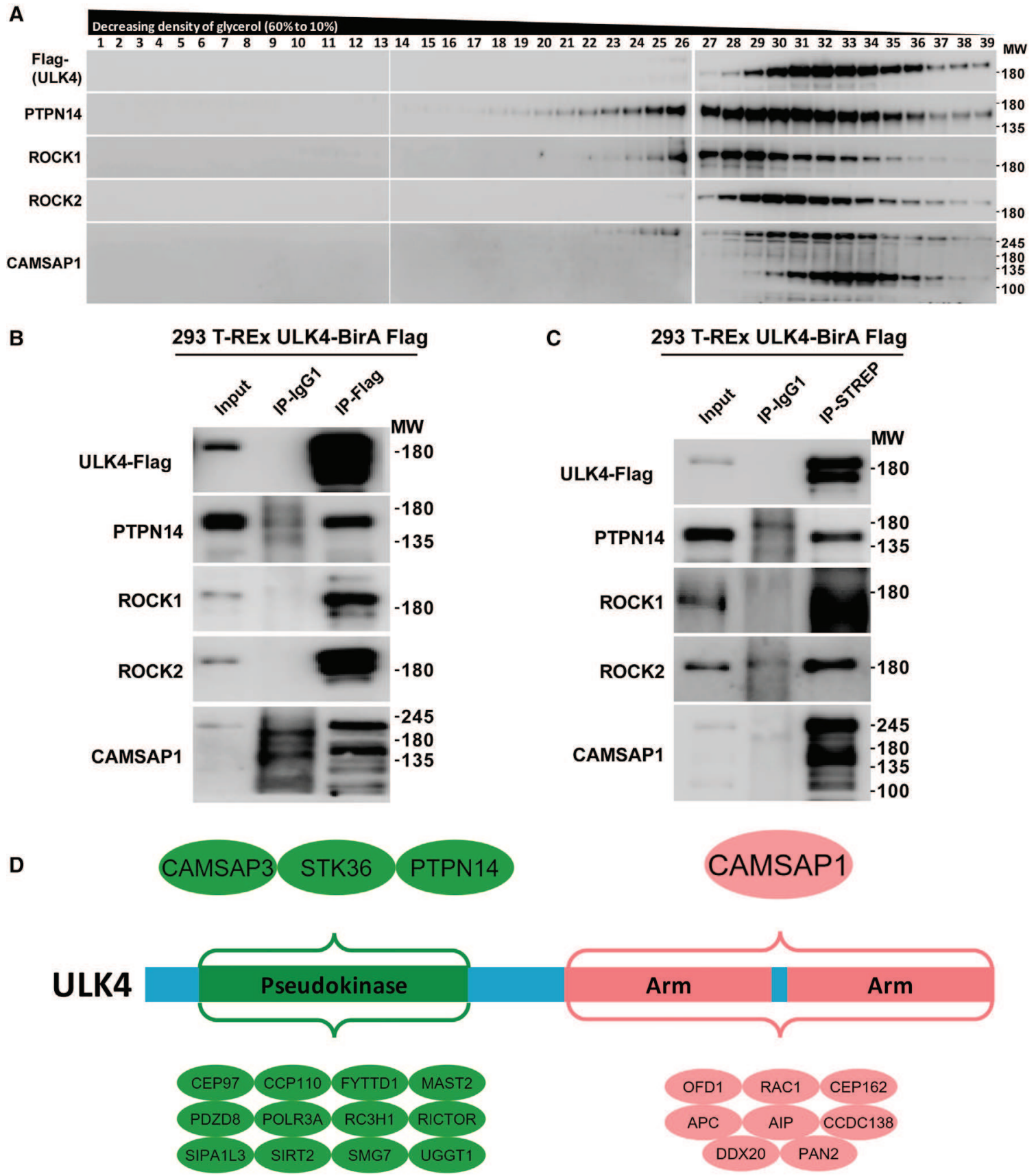

Figure 6. Association Studies Using Density Gradients and Immunoprecipitation

(A) Whole-cell lysate from tetracycline-treated Flp-In T-REx 293 cells expressing C-terminally FLAG-tagged full-length ULK4 (ULK4-BirA*Flag) was used for density gradient ultra-centrifugation. Immunoblot showed that fractions containing ULK4 also contained PTPN14, ROCK1, ROCK2, and CAMSAP1.

(B) ULK4 was immunoprecipitated from tetracycline-treated Flp-In T-REx 293 cells expressing full-length ULK4-BirA*Flag. Subsequent immunoblotting showed co-immunoprecipitation of PTPN14, ROCK1, ROCK2, and CAMSAP1.

(C) Flp-In T-REx 293 cells expressing tetracycline-inducible Flag- and BirA-tagged full-length ULK4 (ULK4-BirA*Flag) were treated with biotin for $24 \mathrm{~h}$. Proteins thus biotinylated were pulled down using streptavidin-conjugated beads. Immunoblot showed the presence of PTPN14, ROCK1, ROCK2, and CAMSAP1 in the eluate.

(D) Domain mapping of ULK4 proximity interacting proteins. Proximity interactors of FlagBirA*-ULK4 and/or ULK4-BirA*Flag pseudokinase domain or armadillo repeat (Arm) domains fusion proteins expressed in Flp-In T-REx 293 cells are shown. Probability of specific interactors was determined using Bayesian statistics against a panel of FlagBirA* only BiolD samples. Proteins with a Bayesian false discovery rate of interaction of $\leq 0.01$ were considered statistically significant putative proximity interactors.

See also Figure S7 and Tables S2 and S3. 


\section{STAR $\star M E T H O D S$}

Detailed methods are provided in the online version of this paper and include the following:

- KEY RESOURCES TABLE

- RESOURCE AVAILABILITY

$\bigcirc$ Lead Contact

- Material Availability

O Data and Code Availability

- EXPERIMENTAL MODEL AND SUBJECT DETAILS

○ Bacterial Cell Culture

○ Mammalian Cell Culture

- METHODS DETAILS

O Cloning

Protein Expression and Purification

$\bigcirc$ Differential Scanning Fluorimetry (DSF)

- Crystallisation of the ULK4 ${ }_{\mathrm{PD}}-\mathrm{ATP} \gamma \mathrm{S}$ Complex

○ BiolD Sample Processing

○ Liquid Chromatography - Mass Spectrometry

- LC-MS Data Processing

O Cloning and Generation of Cell Lines

○ Density Gradient Ultra-Centrifugation

- Antibodies

O Immunoprecipitation

O Immunofluorescence

○ Phylogenetic Tree

O Activation Loop Length Distribution

O Molecular Dynamics (MD) of ULK4 with ATP Bound

O Identification of ULK4 Sequence Constraints

- QUANTIFICATION AND STATISTICAL ANALYSIS

\section{SUPPLEMENTAL INFORMATION}

Supplemental Information can be found online at https://doi.org/10.1016/j.str. 2020.07.016.

\section{ACKNOWLEDGMENTS}

S.K., F.P., D.C., and S.M. are grateful for support by the SGC, a registered charity (no. 1097737) that receives funds from AbbVie, Bayer Pharma AG, Boehringer Ingelheim, Canada Foundation for Innovation, Eshelman Institute for Innovation, Genome Canada, Innovative Medicines Initiative (EU/EFPIA EUbOPEN, grant agreement no. 875510), Janssen, Merck KGaA Germany, MSD, Novartis Pharma AG, Ontario Ministry of Economic Development and Innovation, Pfizer, São Paulo Research Foundation-FAPESP, Takeda, and Wellcome. Funding for N.K. from the NIH (1U01CA239106-01: a data analytics framework for mining the dark kinome) is acknowledged. S.K. is grateful for support by the Collaborative Sonderforschungsbereich 1177 Autophagy (SFB1177) at Frankfurt University, as well as the German Cancer Consortium (DKTK). The data collection at SLS has been supported by funding from the European Union's Horizon 2020 Research and Innovation program under grant agreement no. 730872, project CALIPSOplus.

\section{AUTHOR CONTRIBUTIONS}

S.K., S.M., B.R., and R.R. designed the research. F.P. and D.C. performed protein biochemistry and crystallization. S.M. solved and refined the ULK4 crystal structure. S.S. and N.K. performed the bioinformatics analysis. B.R., J.St-G., and M.S. performed the BiolD study and validation data on interaction partners. S.K., S.M., and N.K. wrote the manuscript, which was approved by all authors.

\section{DECLARATION OF INTERESTS}

The authors declare no competing interests.

Received: April 24, 2020

Revised: June 17, 2020

Accepted: July 29, 2020

Published: August 18, 2020

\section{REFERENCES}

Abraham, M.J., Murtola, T., Schulz, R., Páll, S., Smith, J.C., Hess, B., and Lindahl, E. (2015). GROMACS: high performance molecular simulations through multi-level parallelism from laptops to supercomputers. SoftwareX 1-2, 19-25.

Akhmanova, A., and Hoogenraad, C.C. (2015). Microtubule minus-end-targeting proteins. Curr. Biol. 25, R162-R171.

Allan, J.E., and Doherty, P.C. (1990). Binding of monoclonal antibodies and T cell effector function in vivo. Hybridoma 9, 9-15.

Atherton, J., Luo, Y., Xiang, S., Yang, C., Rai, A., Jiang, K., Stangier, M., Vemu, A., Cook, A.D., Wang, S., et al. (2019). Structural determinants of microtubule minus end preference in CAMSAP CKK domains. Nat. Commun. 10, 5236.

Bailey, F.P., Byrne, D.P., Oruganty, K., Eyers, C.E., Novotny, C.J., Shokat, K.M., Kannan, N., and Eyers, P.A. (2015). The Tribbles 2 (TRB2) pseudokinase binds to ATP and autophosphorylates in a metal-independent manner. Biochem. J. 467, 47-62.

Baines, A.J., Bignone, P.A., King, M.D., Maggs, A.M., Bennett, P.M., Pinder, J.C., and Phillips, G.W. (2009). The CKK domain (DUF1781) binds microtubules and defines the CAMSAP/ssp4 family of animal proteins. Mol. Biol. Evol. 26, 2005-2014.

Berendsen, H.J.C., Postma, J.P.M., van Gunsteren, W.F., DiNola, A., and Haak, J.R. (1984). Molecular dynamics with coupling to an external bath. J. Chem. Phys. 81, 3684.

Berendsen, H.J.C., van der Spoel, D., and van Drunen, R. (1995). GROMACS: a message-passing parallel molecular dynamics implementation. Comput. Phys. Commun. 91, 43-56.

Boudeau, J., Miranda-Saavedra, D., Barton, G.J., and Alessi, D.R. (2006). Emerging roles of pseudokinases. Trends Cell Biol. 16, 443-452.

Broderick, P., Chubb, D., Johnson, D.C., Weinhold, N., Forsti, A., Lloyd, A., Olver, B., Ma, Y., Dobbins, S.E., Walker, B.A., et al. (2011). Common variation at 3p22.1 and 7p15.3 influences multiple myeloma risk. Nat. Genet. 44, 58-61. Burgess-Brown, N.A., Mahajan, P., Strain-Damerell, C., Gileadi, O., and Graslund, S. (2014). Medium-throughput production of recombinant human proteins: protein production in E. coli. Methods Mol. Biol. 1091, 73-94.

Burgess, S.G., Mukherjee, M., Sabir, S., Joseph, N., Gutierrez-Caballero, C., Richards, M.W., Huguenin-Dezot, N., Chin, J.W., Kennedy, E.J., Pfuhl, M., et al. (2018). Mitotic spindle association of TACC3 requires Aurora-A-dependent stabilization of a cryptic alpha-helix. EMBO J. 37, e97902.

Bussi, G., Donadio, D., and Parrinello, M. (2007). Canonical sampling through velocity rescaling. J. Chem. Phys. 126, 014101.

Caballe, A., Wenzel, D.M., Agromayor, M., Alam, S.L., Skalicky, J.J., Kloc, M., Carlton, J.G., Labrador, L., Sundquist, W.I., and Martin-Serrano, J. (2015). ULK3 regulates cytokinetic abscission by phosphorylating ESCRT-III proteins. eLife 4, e06547.

Chaikuad, A., Koschade, S.E., Stolz, A., Zivkovic, K., Pohl, C., Shaid, S., Ren, H., Lambert, L.J., Cosford, N.D.P., Brandts, C.H., et al. (2019). Conservation of structure, function and inhibitor binding in UNC-51-like kinase 1 and 2 (ULK1) 2). Biochem. J. 476, 875-887.

Chambers, M.C., Maclean, B., Burke, R., Amodei, D., Ruderman, D.L., Neumann, S., Gatto, L., Fischer, B., Pratt, B., Egertson, J., et al. (2012). A cross-platform toolkit for mass spectrometry and proteomics. Nat. Biotechnol. 30, 918-920.

Chen, V.B., Arendall, W.B., 3rd, Headd, J.J., Keedy, D.A., Immormino, R.M., Kapral, G.J., Murray, L.W., Richardson, J.S., and Richardson, D.C. (2010). 
MolProbity: all-atom structure validation for macromolecular crystallography. Acta Crystallogr. D Biol. Crystallogr. 66, 12-21.

Choi, H., Larsen, B., Lin, Z.Y., Breitkreutz, A., Mellacheruvu, D., Fermin, D., Qin, Z.S., Tyers, M., Gingras, A.C., and Nesvizhskii, A.I. (2011). SAINT: probabilistic scoring of affinity purification-mass spectrometry data. Nat. Methods 8, 70-73.

Citri, A., Skaria, K.B., and Yarden, Y. (2003). The deaf and the dumb: the biology of ErbB-2 and ErbB-3. Exp. Cell Res. 284, 54-65.

Crooks, G.E., Hon, G., Chandonia, J.M., and Brenner, S.E. (2004). WebLogo: a sequence logo generator. Genome Res. 14, 1188-1190.

Deutsch, E.W., Mendoza, L., Shteynberg, D., Slagel, J., Sun, Z., and Moritz, R.L. (2015). Trans-proteomic pipeline, a standardized data processing pipeline for large-scale reproducible proteomics informatics. Proteomics Clin. Appl. 9, 745-754.

Durzynska, I., Xu, X., Adelmant, G., Ficarro, S.B., Marto, J.A., Sliz, P., Uljon, S., and Blacklow, S.C. (2017). STK40 is a pseudokinase that binds the E3 ubiquitin ligase COP1. Structure 25, 287-294.

Ehret, G.B., Ferreira, T., Chasman, D.I., Jackson, A.U., Schmidt, E.M., Johnson, T., Thorleifsson, G., Luan, J., Donnelly, L.A., Kanoni, S., et al. (2016). The genetics of blood pressure regulation and its target organs from association studies in 342,415 individuals. Nat. Genet. 48, 1171-1184.

Emsley, P., and Cowtan, K. (2004). Coot: model-building tools for molecular graphics. Acta Crystallogr. D Biol. Crystallogr. 60, 2126-2132.

Eng, J.K., Jahan, T.A., and Hoopmann, M.R. (2013). Comet: an open-source MS/MS sequence database search tool. Proteomics 13, 22-24.

Eswaran, J., Lee, W.H., Debreczeni, J.E., Filippakopoulos, P., Turnbull, A., Fedorov, O., Deacon, S.W., Peterson, J.R., and Knapp, S. (2007). Crystal structures of the p21-activated kinases PAK4, PAK5, and PAK6 reveal catalytic domain plasticity of active group II PAKs. Structure 15, 201-213.

Fedorov, O., Niesen, F.H., and Knapp, S. (2012). Kinase inhibitor selectivity profiling using differential scanning fluorimetry. Methods Mol. Biol. 795, 109-118.

Gingras, A.C., Abe, K.T., and Raught, B. (2019). Getting to know the neighborhood: using proximity-dependent biotinylation to characterize protein complexes and map organelles. Curr. Opin. Chem. Biol. 48, 44-54.

Goshima, G., Mayer, M., Zhang, N., Stuurman, N., and Vale, R.D. (2008). Augmin: a protein complex required for centrosome-independent microtubule generation within the spindle. J. Cell Biol. 181, 421-429.

Guo, D.C., Grove, M.L., Prakash, S.K., Eriksson, P., Hostetler, E.M., LeMaire, S.A., Body, S.C., Shalhub, S., Estrera, A.L., Safi, H.J., et al. (2016). Genetic variants in LRP1 and ULK4 are associated with acute aortic dissections. Am. J. Hum. Genet. 99, 762-769.

Gupta, G.D., Coyaud, E., Goncalves, J., Mojarad, B.A., Liu, Y., Wu, Q., Gheiratmand, L., Comartin, D., Tkach, J.M., Cheung, S.W., et al. (2015). A dynamic protein interaction landscape of the human centrosome-cilium interface. Cell 163, 1484-1499.

Ha, B.H., and Boggon, T.J. (2018). The crystal structure of pseudokinase PEAK1 (Sugen kinase 269) reveals an unusual catalytic cleft and a novel mode of kinase fold dimerization. J. Biol. Chem. 293, 1642-1650.

Han, Y., Wang, B., Cho, Y.S., Zhu, J., Wu, J., Chen, Y., and Jiang, J. (2019). Phosphorylation of ci/Gli by fused family kinases promotes hedgehog signaling. Dev. Cell 50, 610-626 e614.

Huang, J., and MacKerell, A.D., Jr. (2013). CHARMM36 all-atom additive protein force field: validation based on comparison to NMR data. J. Comput. Chem. 34, 2135-2145.

Jacobsen, A.V., and Murphy, J.M. (2017). The secret life of kinases: insights into non-catalytic signalling functions from pseudokinases. Biochem. Soc. Trans. 45, 665-681.

Jura, N., Shan, Y., Cao, X., Shaw, D.E., and Kuriyan, J. (2009). Structural analysis of the catalytically inactive kinase domain of the human EGF receptor 3. Proc. Natl. Acad. Sci. U S A 106, 21608-21613.

Kabsch, W., and Sander, C. (1983). Dictionary of protein secondary structure: pattern recognition of hydrogen-bonded and geometrical features. Biopolymers 22, 2577-2637.
Kannan, N., Taylor, S.S., Zhai, Y., Venter, J.C., and Manning, G. (2007). Structural and functional diversity of the microbial kinome. PLoS Biol. 5, e17. Katoh, K., and Standley, D.M. (2013). MAFFT multiple sequence alignment software version 7: improvements in performance and usability. Mol. Biol. Evol. 30, 772-780.

Khamrui, S., Ung, P.M.U., Secor, C., Schlessinger, A., and Lazarus, M.B. (2020). High-resolution structure and inhibition of the schizophrenia-linked pseudokinase ULK4. J. Am. Chem. Soc. 142, 33-37.

Kwon, A., Scott, S., Taujale, R., Yeung, W., Kochut, K.J., Eyers, P.A., and Kannan, N. (2019). Tracing the origin and evolution of pseudokinases across the tree of life. Sci. Signal. 12.

Lang, B., Pu, J., Hunter, I., Liu, M., Martin-Granados, C., Reilly, T.J., Gao, G.D., Guan, Z.L., Li, W.D., Shi, Y.Y., et al. (2014). Recurrent deletions of ULK4 in schizophrenia: a gene crucial for neuritogenesis and neuronal motility. J. Cell Sci. 127, 630-640.

Lang, B., Zhang, L., Jiang, G., Hu, L., Lan, W., Zhao, L., Hunter, I., Pruski, M., Song, N.N., Huang, Y., et al. (2016). Control of cortex development by ULK4, a rare risk gene for mental disorders including schizophrenia. Sci. Rep. 6, 31126. Lazarus, M.B., Novotny, C.J., and Shokat, K.M. (2015). Structure of the human autophagy initiating kinase ULK1 in complex with potent inhibitors. ACS Chem. Biol. 10, 257-261.

Lazarus, M.B., and Shokat, K.M. (2015). Discovery and structure of a new inhibitor scaffold of the autophagy initiating kinase ULK1. Bioorg. Med. Chem. 23, 5483-5488.

Letunic, I., and Bork, P. (2016). Interactive tree of life (iTOL) v3: an online tool for the display and annotation of phylogenetic and other trees. Nucleic Acids Res. 44, W242-W245.

Levy, D., Ehret, G.B., Rice, K., Verwoert, G.C., Launer, L.J., Dehghan, A., Glazer, N.L., Morrison, A.C., Johnson, A.D., Aspelund, T., et al. (2009). Genome-wide association study of blood pressure and hypertension. Nat. Genet. 41, 677-687.

Liu, M., Fitzgibbon, M., Wang, Y., Reilly, J., Qian, X., O’Brien, T., Clapcote, S., Shen, S., and Roche, M. (2018a). Ulk4 regulates GABAergic signaling and anxiety-related behavior. Transl. Psychiatry 8, 43.

Liu, M., Xu, P., Guan, Z., Qian, X., Dockery, P., Fitzgerald, U., O’Brien, T., and Shen, S. (2018b). Ulk4 deficiency leads to hypomyelination in mice. Glia 66, 175-190.

Lucet, I.S., and Murphy, J.M. (2017). Characterization of ligand binding to pseudokinases using a thermal shift assay. Methods Mol. Biol. 1636, 91-104. Lupardus, P.J., Ultsch, M., Wallweber, H., Bir Kohli, P., Johnson, A.R., and Eigenbrot, C. (2014). Structure of the pseudokinase-kinase domains from protein kinase TYK2 reveals a mechanism for Janus kinase (JAK) autoinhibition. Proc. Natl. Acad. Sci. U S A 111, 8025-8030.

Manning, G., Whyte, D.B., Martinez, R., Hunter, T., and Sudarsanam, S. (2002). The protein kinase complement of the human genome. Science 298 1912-1934.

Marcette, J.D., Chen, J.J., and Nonet, M.L. (2014). The Caenorhabditis elegans microtubule minus-end binding homolog PTRN-1 stabilizes synapses and neurites. eLife 3, e01637.

Mathea, S., Salah, E., Moroglu, M., Scorah, A., von Delft, F., Arrowsmith, C.H., Edwards, A.M., Bountra, C., Huber, K., Knapp, S. (2018). Unc-51-Like kinase 3 (ULK3) in complex with bosutinib. PDB Entry - 6FDY, https://doi.org/10.2210/ pdb6fdy/pdb.

McCoy, A.J., Grosse-Kunstleve, R.W., Storoni, L.C., and Read, R.J. (2005). Likelihood-enhanced fast translation functions. Acta Crystallogr. D Biol. Crystallogr. 61, 458-464.

McSkimming, D.I., Dastgheib, S., Baffi, T.R., Byrne, D.P., Ferries, S., Scott, S.T., Newton, A.C., Eyers, C.E., Kochut, K.J., Eyers, P.A., et al. (2016) KinView: a visual comparative sequence analysis tool for integrated kinome research. Mol. Biosyst. 12, 3651-3665.

Mukherjee, K., Sharma, M., Urlaub, H., Bourenkov, G.P., Jahn, R., Sudhof, T.C., and Wahl, M.C. (2008). CASK functions as a $\mathrm{Mg}^{2+}$-independent neurexin kinase. Cell 133, 328-339. 
Murone, M., Luoh, S.M., Stone, D., Li, W., Gurney, A., Armanini, M., Grey, C., Rosenthal, A., and de Sauvage, F.J. (2000). Gli regulation by the opposing activities of fused and suppressor of fused. Nat. Cell Biol. 2, 310-312.

Murphy, J.M., Czabotar, P.E., Hildebrand, J.M., Lucet, I.S., Zhang, J.G., Alvarez-Diaz, S., Lewis, R., Lalaoui, N., Metcalf, D., Webb, A.I., et al. (2013). The pseudokinase MLKL mediates necroptosis via a molecular switch mechanism. Immunity 39, 443-453.

Murphy, J.M., Farhan, H., and Eyers, P.A. (2017). Bio-Zombie: the rise of pseudoenzymes in biology. Biochem. Soc. Trans. 45, 537-544.

Murphy, J.M., Nakatani, Y., Jamieson, S.A., Dai, W., Lucet, I.S., and Mace, P.D. (2015). Molecular mechanism of CCAAT-enhancer binding protein recruitment by the TRIB1 pseudokinase. Structure 23, 2111-2121.

Murphy, J.M., Zhang, Q., Young, S.N., Reese, M.L., Bailey, F.P., Eyers, P.A., Ungureanu, D., Hammaren, H., Silvennoinen, O., Varghese, L.N., et al. (2014). A robust methodology to subclassify pseudokinases based on their nucleotide-binding properties. Biochem. J. 457, 323-334.

Murshudov, G.N., Vagin, A.A., and Dodson, E.J. (1997). Refinement of macromolecular structures by the maximum-likelihood method. Acta Crystallogr. D Biol. Crystallogr. 53, 240-255.

Neuwald, A.F. (2009). Rapid detection, classification and accurate alignment of up to a million or more related protein sequences. Bioinformatics 25, 1869-1875.

Neuwald, A.F. (2014). A Bayesian sampler for optimization of protein domain hierarchies. J. Comput. Biol. 21, 269-286.

Niesen, F.H., Berglund, H., and Vedadi, M. (2007). The use of differential scanning fluorimetry to detect ligand interactions that promote protein stability. Nat. Protoc. 2, 2212-2221.

Oprea, T.I. (2019). Exploring the dark genome: implications for precision medicine. Mamm. Genome 30, 192-200.

Páll, S., and Hess, B. (2013). A flexible algorithm for calculating pair interactions on SIMD architectures. Comput. Phys. Commun. 184, 2641-2650.

Patel, O., Griffin, M.D.W., Panjikar, S., Dai, W., Ma, X., Chan, H., Zheng, C., Kropp, A., Murphy, J.M., Daly, R.J., et al. (2017). Structure of SgK223 pseudokinase reveals novel mechanisms of homotypic and heterotypic association. Nat. Commun. 8, 1157.

Petrie, E.J., Sandow, J.J., Jacobsen, A.V., Smith, B.J., Griffin, M.D.W., Lucet, I.S., Dai, W., Young, S.N., Tanzer, M.C., Wardak, A., et al. (2018). Conformational switching of the pseudokinase domain promotes human MLKL tetramerization and cell death by necroptosis. Nat. Commun. 9, 2422. Pongrakhananon, V., Saito, H., Hiver, S., Abe, T., Shioi, G., Meng, W., and Takeichi, M. (2018). CAMSAP3 maintains neuronal polarity through regulation of microtubule stability. Proc. Natl. Acad. Sci. U S A 115, 9750-9755.

Price, M.N., Dehal, P.S., and Arkin, A.P. (2010). FastTree 2-approximately maximum-likelihood trees for large alignments. PLoS One 5, e9490.

R Core Team (2020). R: A Language and Environment for Statistical Computing, Version 3.6.3. https://www.R-project.org.

Ribeiro, A.J.M., Das, S., Dawson, N., Zaru, R., Orchard, S., Thornton, J.M., Orengo, C., Zeqiraj, E., Murphy, J.M., and Eyers, P.A. (2019). Emerging con- cepts in pseudoenzyme classification, evolution, and signaling. Sci. Signal. 12, eaat9797.

Roux, K.J. (2013). Marked by association: techniques for proximity-dependent labeling of proteins in eukaryotic cells. Cell Mol Life Sci. 70, 3657-3664.

Scheeff, E.D., Eswaran, J., Bunkoczi, G., Knapp, S., and Manning, G. (2009). Structure of the pseudokinase VRK3 reveals a degraded catalytic site, a highly conserved kinase fold, and a putative regulatory binding site. Structure 17, 128-138.

Shrestha, S., Byrne, D.P., Harris, J.A., Kannan, N., and Eyers, P.A. (2020). Cataloguing the dead: breathing new life into pseudokinase research. FEBS J. https://doi.org/10.1111/febs.15246.

Sun, L., Wang, H., Wang, Z., He, S., Chen, S., Liao, D., Wang, L., Yan, J., Liu, W., Lei, X., et al. (2012). Mixed lineage kinase domain-like protein mediates necrosis signaling downstream of RIP3 kinase. Cell 148, 213-227.

Talevich, E., Mirza, A., and Kannan, N. (2011). Structural and evolutionary divergence of eukaryotic protein kinases in Apicomplexa. BMC Evol. Biol. $11,321$.

Teo, G., Liu, G., Zhang, J., Nesvizhskii, A.I., Gingras, A.C., and Choi, H. (2014). SAINTexpress: improvements and additional features in Significance Analysis of INTeractome software. J. Proteomics 100, 37-43.

Uehara, R., Nozawa, R.S., Tomioka, A., Petry, S., Vale, R.D., Obuse, C., and Goshima, G. (2009). The augmin complex plays a critical role in spindle microtubule generation for mitotic progression and cytokinesis in human cells. Proc. Natl. Acad. Sci. U S A 106, 6998-7003.

Vogel, P., Read, R.W., Hansen, G.M., Payne, B.J., Small, D., Sands, A.T., and Zambrowicz, B.P. (2012). Congenital hydrocephalus in genetically engineered mice. Vet. Pathol. 49, 166-181.

Vriend, G. (1990). What IF: a molecular modeling and drug design program. J. Mol. Graph. 8, 52-56.

Wang, C., Bradley, P., and Baker, D. (2007). Protein-protein docking with backbone flexibility. J. Mol. Biol. 373, 503-519.

Wang, H., Sun, L., Su, L., Rizo, J., Liu, L., Wang, L.F., Wang, F.S., and Wang, X. (2014). Mixed lineage kinase domain-like protein MLKL causes necrotic membrane disruption upon phosphorylation by RIP3. Mol. Cell 54, 133-146.

Winter, G. (2010). xia2: an expert system for macromolecular crystallography data reduction. J. Appl. Crystallogr. 43, 186-190.

Zachari, M., and Ganley, I.G. (2017). The mammalian ULK1 complex and autophagy initiation. Essays Biochem. 61, 585-596.

Zeqiraj, E., Filippi, B.M., Deak, M., Alessi, D.R., and van Aalten, D.M. (2009a). Structure of the LKB1-STRAD-MO25 complex reveals an allosteric mechanism of kinase activation. Science 326, 1707-1711.

Zeqiraj, E., Filippi, B.M., Goldie, S., Navratilova, I., Boudeau, J., Deak, M., Alessi, D.R., and van Aalten, D.M. (2009b). ATP and MO25alpha regulate the conformational state of the STRADalpha pseudokinase and activation of the LKB1 tumour suppressor. Plos Biol. 7, e1000126.

Zheng, J., Trafny, E.A., Knighton, D.R., Xuong, N.H., Taylor, S.S., Ten Eyck, L.F., and Sowadski, J.M. (1993). 2.2 A refined crystal structure of the catalytic subunit of cAMP-dependent protein kinase complexed with MnATP and a peptide inhibitor. Acta Crystallogr. D Biol. Crystallogr. 49, 362-365. 


\section{STAR $\star$ METHODS}

\section{KEY RESOURCES TABLE}

\begin{tabular}{|c|c|c|}
\hline REAGENT or RESOURCE & SOURCE & IDENTIFIER \\
\hline \multicolumn{3}{|l|}{ Antibodies } \\
\hline Anti-Flag & Cell Signaling Technology & Cat\#14793; RRID: AB_2572291 \\
\hline Anti-PTPN14 & Cell Signaling Technology & Cat\#13808 ; RRID: AB_2798318 \\
\hline Anti-ROCK1 & Cell Signaling Technology & Cat\#4035; RRID: AB_2238679 \\
\hline Anti-ROCK2 & Cell Signaling Technology & Cat\#9029; RRID: AB_11127802 \\
\hline Anti-CAMSAP1 & Abcam & Cat\#86000; RRID: AB_1924847 \\
\hline Texas Red goat anti-rabbit & ThermoFisher Scientific & Cat\#T6391; RRID: AB_10374713 \\
\hline \multicolumn{3}{|l|}{ Bacterial and Virus Strains } \\
\hline Escherichia coli Rosetta & Novagen & Cat\#70954 \\
\hline \multicolumn{3}{|l|}{ Chemicals, Peptides, and Recombinant Proteins } \\
\hline HEPES & Fisher BioReagents & Cat\#BP310-1 \\
\hline $\mathrm{NaCl}$ & Fisher BioReagents & Cat\#S/3160/65 \\
\hline TCEP & Goldbio & Cat\#TCEP25 \\
\hline Imidazole & Alfa Aesar & Cat\#A10221 \\
\hline IPTG & Europa Bioproducts Ltd. & Cat\#IN102A \\
\hline DMEM cell culture medium & Wisent Bio Products & Cat\#319-005-CL \\
\hline Fetal Bovine serum (FBS) & Wisent Bio Products & Cat\#081-150 \\
\hline ZeocinTm & ThermoFisher & Cat\#R25005 \\
\hline Penicillin-Streptomycin Solution & Wisent Bio Products & Cat\# 450-201-EL \\
\hline Blasticidin S & BioShop & Cat\#BLA500 \\
\hline Hygromycin B & BioShop & Cat\# 31282-04-9 \\
\hline LipoD293TM & Signagen & Cat\#SL100668 \\
\hline Terrific broth & Merck Millipore & Cat\#101629 \\
\hline Glycerol & Fisher BioReagents & Cat\#G/0650/17 \\
\hline PEG4K & Molecular dimensions & N/A \\
\hline ATP $\gamma \mathrm{S}$ & Jena Bioscience & Cat\#NU-406-5 \\
\hline citrate & Molecular dimensions & N/A \\
\hline 2-propanol & Molecular dimensions & $\mathrm{N} / \mathrm{A}$ \\
\hline Ethylene glycol & Fluka Analytical & Cat\#03750 \\
\hline SYPRO orange & Sigma & Cat\#S5692 \\
\hline LipoD293 & SignaGen Laboratories & Cat\#SL100668 \\
\hline CHAPS & MilliporeSigma & Cat\#C3023 \\
\hline tetracycline & MilliporeSigma & Cat\#T3383 \\
\hline Biotin & Biobasic & Cat\#BB0078 \\
\hline \multicolumn{3}{|l|}{ Deposited Data } \\
\hline ULK4 in complex with ATPgammaS & This paper & PDB: $6 \mathrm{TSZ}$ \\
\hline Structure of human ULK4 in complex with inhibitor & Khamrui et al., 2020 & PDB: $6 U 5 \mathrm{~L}$ \\
\hline Structure of ULK1 bound to an inhibitor & Lazarus et al., 2015 & PDB: 4WNO \\
\hline $\begin{array}{l}\text { Pseudokinase ad C-terminal extension of } \\
\text { Human Tribbles Homolog } 1\end{array}$ & Murphy et al., 2015 & PDB: 5CEM \\
\hline Structure of MLKL & Murphy et al., 2013 & PDB: 4BTF \\
\hline $\begin{array}{l}\text { 2.2 A refined crystal structure of the catalytic } \\
\text { subunit of cAMP-dependent protein kinase } \\
\text { complexed with MNATP and a peptide inhibitor }\end{array}$ & Zheng et al., 1993 & PDB: 1ATP \\
\hline Structure of STRAD and MO25 & Zeqiraj et al., 2009a, 2009b & PDB: 3GNI \\
\hline Unc-51-Like Kinase 3 (ULK3) In Complex With Bosutinib & Mathea et al., 2018 & PDB: 6FDY \\
\hline
\end{tabular}




\begin{tabular}{|c|c|c|}
\hline Continued & & \\
\hline REAGENT or RESOURCE & SOURCE & IDENTIFIER \\
\hline Structure of ULK1 bound to a selective inhibitor & Lazarus and Shokat, 2015 & PDB: $5 \mathrm{Cl} 7$ \\
\hline Crystal structure of ULK2 in complexed with hesperadin & Chaikuad et al., 2019 & PDB: 6QAT \\
\hline Mass spectrometry data & This paper & $\begin{array}{l}\text { ID MSV000084747 } \\
\text { massive.ucsd.edu }\end{array}$ \\
\hline \multicolumn{3}{|l|}{ Experimental Models: Cell Lines } \\
\hline Human: Flp-In T-REx 293 cells & Thermo Fisher & Cat\#R780-07 \\
\hline \multicolumn{3}{|l|}{ Oligonucleotides } \\
\hline $\begin{array}{l}\text { Mutagenesis forward primer for ULK4 K39R: } \\
\text { GTGCACCGATAAGTGCAGACGTCCGGAGATTACCAACTG }\end{array}$ & Eurofins & N/A \\
\hline $\begin{array}{l}\text { Mutagenesis reverse primer for ULK4 K39R: } \\
\text { CAGTTGGTAATCTCCGGACGTCTGCACTTATCGGTGCAC }\end{array}$ & Eurofins & $\mathrm{N} / \mathrm{A}$ \\
\hline $\begin{array}{l}\text { Mutagenesis forward primer for ULK4 N139L: } \\
\text { GTACCCTGAAGTTCAGCCTCTTTTGCCTGGCGAAAGTG }\end{array}$ & Eurofins & $\mathrm{N} / \mathrm{A}$ \\
\hline $\begin{array}{l}\text { Mutagenesis reverse primer for ULK4 N139L: } \\
\text { CACTTCGCCAGGCAAAAGAGGCTGAACTTCAGGGTAC }\end{array}$ & Eurofins & N/A \\
\hline \multicolumn{3}{|l|}{ Recombinant DNA } \\
\hline pET-28a(+) encoding ULK4 residues 2-288 & Genscript & $\mathrm{N} / \mathrm{A}$ \\
\hline pET-28a(+) encoding ULK4 K39R residues 2-288 & Genscript & N/A \\
\hline pcDNA3.1-eGFP encoding fullength ULK4 & Genscript & ClonelD\#OHu10418 \\
\hline pcDNA5/FRT/TO encoding full-length ULK4 & This paper & N/A \\
\hline pcDNA5/FRT/TO encoding ULK4 pseudokinase domain & This paper & N/A \\
\hline pcDNA5/FRT/TO encoding ULK4 armadillo repeat domain & This paper & N/A \\
\hline pcDNA5/FRT/TO encoding ULK4 K39R & This paper & N/A \\
\hline \multicolumn{3}{|l|}{ Software and Algorithms } \\
\hline MxPro software & Stratagene & https://www.agilent.com/ \\
\hline FluoView software & Olympus & $\begin{array}{l}\text { https://www.olympus- } \\
\text { lifescience.com/ }\end{array}$ \\
\hline PyMOL (2.3.2) & $\begin{array}{l}\text { The PyMOL Molecular } \\
\text { Graphics System, } \\
\text { Schrödinger, LLC }\end{array}$ & https://pymol.org/2/ \\
\hline Xia2 & Winter, 2010 & https://www.ccp4.ac.uk/ \\
\hline Phaser & McCoy et al., 2005 & https://www.ccp4.ac.uk/ \\
\hline Coot & Emsley and Cowtan, 2004 & https://www.ccp4.ac.uk/ \\
\hline Refmac5 & Murshudov et al., 1997 & https://www.ccp4.ac.uk/ \\
\hline MolProbity & Chen et al., 2010 & molprobity.biochem.duke.edu \\
\hline Proteowizard (v3.0.19311) & Chambers et al., 2012 & http://proteowizard.sourceforge.net/ \\
\hline X!Tandem (v2013.06.15.1) & $\begin{array}{l}\text { The Global Proteome } \\
\text { Machine Organization }\end{array}$ & https://www.thegpm.org/TANDEM/ \\
\hline Comet (2014.02 rev. 2) & Eng et al., 2013 & $\begin{array}{l}\text { https://sourceforge.net/ } \\
\text { projects/comet-ms/ }\end{array}$ \\
\hline Trans-proteomic pipeline (TPP v4.7) & Deutsch et al., 2015 & $\begin{array}{l}\text { http://tools.proteomecenter.org/ } \\
\text { wiki/index.php?title=Software:TPP }\end{array}$ \\
\hline SAINT (v2.5.0) & Choi et al., 2011 & https://omictools.com/saint-tool \\
\hline Gromacs 2018.1 & Berendsen et al., 1995 & $\begin{array}{l}\text { http://www.gromacs.org/ } \\
\text { Downloads }\end{array}$ \\
\hline MAFFT V7.450 & Katoh and Standley, 2013 & $\begin{array}{l}\text { https://mafft.cbrc.jp/ } \\
\text { alignment/software/ }\end{array}$ \\
\hline R version 3.6.3 & R Core Team, 2020 & https://www.r-project.org/ \\
\hline FastTree 2.1 & Price et al., 2010 & $\begin{array}{l}\text { http://www.microbesonline.org/ } \\
\text { fasttree/ }\end{array}$ \\
\hline MAPGAPS 1.0.1 & Neuwald, 2009 & http://mapgaps.igs.umaryland.edu/ \\
\hline
\end{tabular}




\begin{tabular}{|c|c|c|}
\hline Continued & & \\
\hline REAGENT or RESOURCE & SOURCE & IDENTIFIER \\
\hline OmcBPPS 1.0 & Neuwald, 2014 & $\begin{array}{l}\text { http://www.chain.umaryland.edu/ } \\
\text { omcbpps/ }\end{array}$ \\
\hline WebLogo & Crooks et al., 2004 & http://weblogo.berkeley.edu/ \\
\hline \multicolumn{3}{|l|}{ Other } \\
\hline Mx3005P qPCR system & Stratagene & https://www.agilent.com/ \\
\hline 3 Lens crystallisation plate & SWISSCI & 3W96T-PS \\
\hline In-fusion HD cloning kit & Clontech & Cat $\# 639648$ \\
\hline $\begin{array}{l}\text { Inverted microscope with } 60 x / 1.4 \\
\text { PlanApo oil-immersion objective }\end{array}$ & Olympus & IX81 \\
\hline Streptavidin-sepharose beads & GE Healthcare & N/A \\
\hline C18 Acclaim PepMap ${ }^{\text {TM }} 100$ & Thermo Scientific & $\mathrm{N} / \mathrm{A}$ \\
\hline Q-Exactive HF mass spectrometer & Thermo Scientific & N/A \\
\hline DMEM & Wisent & 319-005-CL \\
\hline Tetracycline-free FBS & Wisent & $081-150$ \\
\hline Penicilin-Strreptomycin & Wisent & 450-201-EL \\
\hline Zeocin & ThermoFisher & $\mathrm{R} 25001$ \\
\hline Blasticidin & BioShop & BLA477.25 \\
\hline pcDNA 5/FRT/TO & Thermo Fisher & V6520-20 \\
\hline pOG44 & Thermo Fisher & V600520 \\
\hline LipoD293 & SignaGen & SL100668 \\
\hline Hygromycin B & BioShop & HYG002.1 \\
\hline Tetracycline & Sigma & $\mathrm{T}-3383$ \\
\hline Biotin & Bio Basic & BB0078 \\
\hline Flp-In T-REx 293 & Thermo Fisher & R78007 \\
\hline
\end{tabular}

\section{RESOURCE AVAILABILITY}

\section{Lead Contact}

Further information and requests for resources and reagents should be directed to and will be fulfilled by the Lead Contact, Stefan Knapp (knapp@pharmchem.uni-frankfurt.de)

\section{Material Availability}

Plasmids generated in this study are available from the Lead Contact upon request.

\section{Data and Code Availability}

The model and structure factors reported in this study have been deposited in the PDB database under accession code 6TSZ. All raw mass spectrometry data have been deposited in the MassIVE repository (massive.ucsd.edu) with accession ID MSV000084747.

\section{EXPERIMENTAL MODEL AND SUBJECT DETAILS}

\section{Bacterial Cell Culture}

Recombinant proteins were expressed using Rosetta (DE3) E. coli cells (Novagen) carrying plasmids encoding ULK4 residues 2-288, as listed in the Key Resources Table. Expression was performed in accordance to previously reported methods. (Burgess-Brown et al., 2014). E. coli cultures were grown in Terrific broth medium at $37^{\circ} \mathrm{C}$ till an OD of 1 was reached. The temperature was reduced to $18^{\circ} \mathrm{C}$ and protein expression was induced after 30 min incubation using IPTG. After incubation overnight at $18^{\circ} \mathrm{C}$ cells were harvested by centrifugation (4000 rpm, $\left.15 \mathrm{~min}, 4^{\circ} \mathrm{C}\right)$.

\section{Mammalian Cell Culture}

The female human embryonic kidney Flp-In T-REx 293 cell line containing an inducible expression system (ThermoFisher) was maintained in DMEM (Wisent) supplemented with $10 \%$ tetracycline free fetal bovine serum (Wisent), Penicillin-Streptomycin (Wisent), and $100 \mu \mathrm{g} / \mathrm{mL}$ Zeocin (ThermoFisher) and $5 \mu \mathrm{g} / \mathrm{mL}$ blasticidin (BioShop). For stable transfection, T-REx 293 cells in six well plates were treated with a mixture of plasmids $(0.1 \mu \mathrm{g}$ pcDNA5/FRT/TO containing full length ULK4, kinase domain of ULK4, armadillo repeat domain of ULK4 or ULK4 K39R and $0.9 \mu \mathrm{g}$ pOG44) and LipoD293 transfection reagent according to the manufacturer's instructions 
(Signagen Laboratories). After $18 \mathrm{~h}$, the media was replaced with DMEM, 10\% fetal bovine serum (tetracycline free) and PenicillinStreptomycin. After $24 \mathrm{~h}$ the cells in each well were replated in $10 \mathrm{~cm}$ dishes with DMEM, 10\% fetal bovine serum (tetracycline free), Penicillin-Streptomycin, $200 \mu \mathrm{g} / \mathrm{mL}$ hygromycin B (BioShop) and $5 \mu \mathrm{g} / \mathrm{mL}$ blasticidin (BioShop) allowing for the selection of cells with stably integrated pcDNA 5/FRT/TO ULK4 plasmids. Stably transfected cells grew up in 2 to 3 weeks. Expression and biotinylation of the ULK4 constructs was induced by the addition of $1.0 \mu \mathrm{g} / \mathrm{mL}$ tetracyclin (Sigma) and $50 \mu \mathrm{M}$ biotin (Bio Basic) to the cell medium. The time of cell induction was performed for $24 \mathrm{~h}$ with regard to western blot analysis.

\section{METHODS DETAILS}

\section{Cloning}

The DNA coding for a $\mathrm{His}_{6}$-tag, a TEV cleavage site and the ULK4 residues 2 to 288 was synthesized (Genscript) and cloned into the expression vector pET-28a, using the Ncol and Xhol restriction sites. From this DNA template, the mutants K39R and N139L were generated by site-directed mutagenesis using the QuikChange kit (Agilent).

\section{Protein Expression and Purification}

The expression plasmid was transformed into Rosetta (DE3) competent E. coli (Novagen). The expression was performed as described (Burgess-Brown et al., 2014). Transformed bacteria were grown in TB (Terrific broth, Merck Millipore) and were induced by IPTG (Europa Bioproducts Ltd.) at $\mathrm{OD}_{600}$ of 1.0 after temperature was lowered to 20 degrees $\mathrm{C}$. Cells were harvested after $8 \mathrm{~h}$ induction by centrifugation. Lysis was performed using sonication. For ULK4 $4_{\mathrm{PD}}$ purification, bacteria were re-suspended in lysis buffer ( $50 \mathrm{mM}$ HEPES pH 7.4, $500 \mathrm{mM} \mathrm{NaCl}, 20 \mathrm{mM}$ imidazole, $0.5 \mathrm{mM}$ TCEP, $5 \%$ glycerol) and lysed by sonication (35\% amplitude, $10 \mathrm{~s}$ pulse and $10 \mathrm{~s}$ pause during a $20 \mathrm{~min}$ pulse sequence). The lysate was cleared by centrifugation and loaded onto a Ni NTA column. After vigorous rinsing with lysis buffer the $\mathrm{His}_{6}$-tagged protein was eluted in lysis buffer containing $300 \mathrm{mM}$ imidazole. Finally, ULK4 $4_{P D}$ was concentrated and subjected to gel filtration using an AKTA Xpress system combined with an S200 gel filtration column. The elution volume $91.2 \mathrm{~mL}$ indicated the protein to be monomeric in solution. The final yield was $10 \mathrm{mg}$ ULK4 $\mathrm{PD} / \mathrm{L} \mathrm{TB}$ medium.

\section{Differential Scanning Fluorimetry (DSF)}

The DSF assay of ULK4 against a set of nucleotides was performed according to a previously established protocol (Niesen et al., 2007). A solution of $2 \mu \mathrm{M}$ ULK4 ${ }_{\mathrm{PD}}$ in assay buffer (20 mM HEPES pH 7.4, $150 \mathrm{mM} \mathrm{NaCl}, 0.5 \mathrm{mM}$ TCEP, $5 \%$ glycerol) was mixed 1:1000 with SYPRO Orange (Sigma). The nucleotides to be tested were added to a final concentration of $1 \mathrm{mM}$. $20 \mu \mathrm{L}$ of each sample were placed in a 96 -well plate and heated gradually from $25^{\circ} \mathrm{C}$ to $96^{\circ} \mathrm{C}$. The fluorescence intensity was monitored using an $\mathrm{Mx} 3005 \mathrm{P}$ real-time PCR instrument (Stratagene) with excitation and emission filters set to 465 and $590 \mathrm{~nm}$, respectively. Data was analysed with the MxPro software.

\section{Crystallisation of the ULK4 $4_{\mathrm{PD}}-\mathrm{ATP} \gamma \mathrm{S}$ Complex}

$200 \mathrm{~nL}$ of a solution containing the protein-ligand complex (12 mg/mL ULK4 $\left.4_{\mathrm{PD}}, 1 \mathrm{mM} \mathrm{ATP \gamma S}\right)$ were transferred to a 3-well crystallisation plate (SwisSCl), mixed with $100 \mathrm{~nL}$ precipitant solution ( $0.1 \mathrm{M}$ citrate $\mathrm{pH}$ 5.9, 15\% 2-propanol, 6\% PEG4K) and incubated at $4{ }^{\circ} \mathrm{C}$. Crystals appeared after 2 days and did not change appearance after 7 days. They were mounted in precipitant solution cryoprotected with additional $25 \%$ ethylene glycol. Data was collected at Swiss Light Source, analyzed, scaled and merged with Xia2

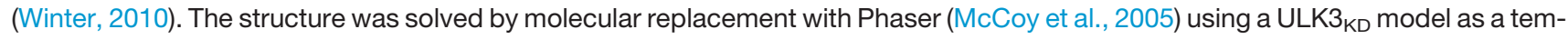
plate (PDB ID 6FDY) and refined by iterative model building using the software Coot (Emsley and Cowtan, 2004) and Refmac5 (Murshudov et al., 1997). The model was validated using MolProbity (Chen et al., 2010). The model and the structure factors have been deposited to the protein databank (http://www.rcsb.org/) with the PDB 6TSZ (crystallographic data collection and refinement data are summarized in Table 1).

\section{BiolD Sample Processing}

BiolD was carried out as reported previously (Gupta et al., 2015). The lysis buffer (50 mM Tris-HCl pH 7.5, 150 mM NaCl, 1 mM EDTA, 1 mM EGTA, $1 \%$ Triton X-100, 0.1\% SDS, protease inhibitor cocktail, turbonuclease) was added to frozen cell pellets, incubated with gentle agitation at $4^{\circ} \mathrm{C}$ for $1 \mathrm{hr}$, briefly sonicated and centrifuged at $16,000 \times \mathrm{g}$ for $30 \mathrm{~min}$ at $4^{\circ} \mathrm{C}$. Supernatants were incubated with $30 \mu \mathrm{L}$ streptavidin-sepharose beads (GE Healthcare) for $3 \mathrm{~h}$ at $4^{\circ} \mathrm{C}$ with gentle agitation. Beads were washed with $\mathrm{NH}_{4} \mathrm{HCO}_{3}(50 \mathrm{mM})$ prior to overnight digestion with MS-grade, TPCK-treated trypsin $\left(1 \mu \mathrm{g}\right.$, Promega) at $37^{\circ} \mathrm{C}$. Additional trypsin $(0.5 \mu \mathrm{g})$ was added, and beads were incubated for $2 \mathrm{hrs}$ at $37^{\circ} \mathrm{C}$. Supernatants were collected and beads rinsed with $\mathrm{NH}_{4} \mathrm{HCO}_{3}(50 \mathrm{mM})$. Both fractions were pooled and samples were lyophilized. Samples were reconstituted in $\mathrm{HCOOH}(0.1 \%)$, de-salted on $\mathrm{C} 18$ columns and lyophilized.

\section{Liquid Chromatography - Mass Spectrometry}

Samples were reconstituted in $\mathrm{HCOOH}(0.1 \%)$, loaded on a pre-column (C18 Acclaim PepMap ${ }^{\mathrm{TM}} 100,75 \mu \mathrm{m} \times 2 \mathrm{~cm}, 3 \mu \mathrm{m}, 100 \AA$, Thermo Scientific) and separated on an analytical column (C18 Acclaim PepMap ${ }^{\mathrm{TM}}$ RSLC, $75 \mu \mathrm{m} \times 50 \mathrm{~cm}, 3 \mathrm{~mm}, 100 \AA$, Thermo Scientific) via high performance liquid chromatography (LC) over a 120 -minute, reversed-phase gradient $\left(5-30 \% \mathrm{CH}_{3} \mathrm{CN}\right.$ in $\left.0.1 \% \mathrm{HCOOH}\right)$ running at $250 \mathrm{nl} / \mathrm{min}$ on an EASY-nLC1000 pump in-line with a Q-Exactive HF mass spectrometer (Thermo Scientific) operated in 
positive ion mode ESI. An MS1 ion scan was performed at 60,000 FWHM followed by MS/MS scans (HCD, 15,000 FWHM) of the twenty most intense parent ions (minimum ion count of 1000 for activation). Dynamic exclusion (within 10 ppm) was set for 5 seconds.

\section{LC-MS Data Processing}

Raw files (.raw) were converted to .mzML format using Proteowizard (v3.0.19311), then searched using X!Tandem (v2013.06.15.1) and Comet (2014.02 rev. 2) against Human RefSeqV104 (containing 36,113 entries). Search parameters specified a parent MS tolerance of $15 \mathrm{ppm}$ and an MS/MS fragment ion tolerance of $0.4 \mathrm{Da}$, with up to two missed cleavages allowed for trypsin. No fixed modifications were set but deamidation (NQ), oxidation (M), acetylation (protein N-term) and diglycine (K) were set as variable modifications. Search results were processed through the trans-proteomic pipeline (TPP v4.7) and proteins to which $\geq 2$ unique peptides were assigned and an iProphet probability $\geq 0.9$ were considered to be high confidence identifications. For statistical analysis, a Bayesian FDR was assigned to identified proteins using SAINT (v2.5.0; 18 BiolD controls compressed to 4). All raw mass spectrometry data have been deposited in the MassIVE repository (massive.ucsd.edu) with accession ID MSV000084747.

\section{Cloning and Generation of Cell Lines}

The following were cloned into pcDNA5/FRT/TO plasmid using in-fusion HD cloning kit (Clontech): full length ULK4, kinase domain of ULK4, armadillo repeat domain of ULK4, ULK4 with point mutation K39R. Each of the inserts was tagged to Flag and BirA either $\mathrm{N}$-terminally or C-terminally. These plasmids were transfected into Flp-In T-REx 293 cells (Thermo Fisher) using LipoD293 reagent (SignaGen Laboratories), and stable expression cell pools were generated. Cells were induced using $1 \mu \mathrm{g} / \mathrm{ml}$ tetracycline. For experiments involving biotinylation, biotin was used at a concentration of $50 \mu \mathrm{M}$.

\section{Density Gradient Ultra-Centrifugation}

Flp-In T-REx 293 cells expressing full length ULK4 (upon induction with tetracycline) were lysed in Tris-buffer containing $0.3 \%$ CHAPS. Cleared whole cell lysate was carefully layered on top of a continuous glycerol gradient (10\%-60\%) in Tris buffer. Following ultra-centrifugation at 35000 r.p.m. for $18 \mathrm{hrs}$ at $4^{\circ} \mathrm{C}$, fractions were collected, boiled with sample buffer and run on SDS-PAGE for subsequent western blot analysis.

\section{Antibodies}

Antibodies against Flag tag, PTPN14, ROCK1 and ROCK2 were procured form Cell Signaling Technology, and that against CAMSAP1 were procured form Abcam.

\section{Immunoprecipitation}

FIp-In T-REx 293 cells expressing full length ULK4 (upon induction with tetracycline) were lysed in Tris-buffer containing $0.3 \%$ CHAPS. Whole cell lysate was subjected to pre-clearing, following which it was used for immunoprecipitation using anti-Flag antibody conjugated to agarose beads. For analysis of biotinylated proteins, streptavidin-conjugated beads were used. The eluate in each case was boiled in sample buffer for immunoblotting.

\section{Immunofluorescence}

FIp-In T-REx 293 cells expressing full length ULK4 (upon induction with tetracycline) was used for staining with anti-Flag antibody. Texas red was used for secondary staining. Confocal imaging was performed with an Olympus IX81 inverted microscope using 60x/1.4 PlanApo oil-immersion objective and FluoView software.

\section{Phylogenetic Tree}

Kinase domains of ULK4 orthologs were aligned with MAFFT V7.450 (Katoh and Standley, 2013). Maximum likelihood tree was generated using FastTree 2.1 (Price et al., 2010). The tree was annotated and visualized using Interactive Tree of Life (ITOL) webserver (Letunic and Bork, 2016).

\section{Activation Loop Length Distribution}

UniProt sequences of ULK4 orthologs were identified using a previously curated eukaryotic Protein Kinase (ePK) profile (Kannan et al., 2007; McSkimming et al., 2016; Talevich et al., 2011) using the MAPGAPS tool (Neuwald, 2009). Residues corresponding to the DFG-Asp (N139 in ULK4) and APE-Glu (E189 in ULK4, Uniprot ID: Q96C45) were used as activation loop boundaries. The lengths of the activation loops were then determined for all ULK4 orthologs and the length distribution was plotted using $R$ version 3.6.3 (R Core Team, 2020).

\section{Molecular Dynamics (MD) of ULK4 with ATP Bound}

Unbiased full atom MD simulation of ULK4 was performed on the solved crystal structure using GROMACS 2018.1 software (Abraham et al., 2015). Residues with ambiguous electron density were modeled using the Whatif server (Vriend, 1990). Main chain atoms for the missing G163 were modeled using RosettaLoop (Wang et al., 2007) using the cyclic coordinate descent (CCD) protocol. Due to the lack reliable force field parameters for ATP $\gamma \mathrm{S}$, we modeled ATP using ATP $\gamma \mathrm{S}$ coordinates as template and performed unrestrained simulations on the ATP bound complex. Both the protein and ATP were parameterized with CHARMM36-March2019 
forcefield (Huang and MacKerell, 2013). The protein was solvated with TIP3P water model in a dodecahedron box. In order to neutralize the charge on the protein, sodium and chloride ions were added to the system. Verlet cutoff was used to define neighbor list for non-bonded interactions (Páll and Hess, 2013). Particle Mesh Ewald (PME) was used to calculate long-range interactions. Energy minimization was performed with steepest-descent algorithm and then with conjugate descent with Fmax less than $500 \mathrm{kJmol}-1 \mathrm{~nm}-1$. The canonical ensemble was carried out by heating the system from $0 \mathrm{~K}$ to $310 \mathrm{~K}$, using velocity rescaling for 100 ps (Bussi et al., 2007). The isothermal-isobaric ensemble ( $P=1$ bar, $T=310 \mathrm{~K}$ ) was carried out using the Berendsen barostat for 100 ps (Berendsen et al., 1984). The unrestrained MD productions were collected using a time step of 2 fs after the isothermal-isobaric ensemble. The trajectories were processed and analyzed using the GROMACS built-in tools. Secondary structures for the MD trajectory was defined using DSSP (Allan and Doherty, 1990; Kabsch and Sander, 1983). Structural visualization was performed using PyMOL [PyMOL 2.3.2]. Snapshots of the MD simulation of ULK4 with ATP bound are shown in Figure S5. A movie illustrating the MD simulation is available as Video $\mathrm{S} 1$.

\section{Identification of ULK4 Sequence Constraints}

ULK1-4 UniProt sequences were identified and aligned using previously curated hierarchical ePK profiles (See STAR Methods, Activation loop length distribution). Residues distinguishing ULK4 sequences from other ULK paralogs were then identified using the optimal multiple category Bayesian Partitioning with Pattern Selection (omcBPPS) program (Neuwald, 2014).

\section{QUANTIFICATION AND STATISTICAL ANALYSIS}

Crystallographic data processing was performed using a variety of programs of the CCP4 software suite as described in the "Crystallisation of the ULK4PD-ATP S Complex" section of the Method Details. Xia2 was used for the analysis, scaling and merging of data. Phaser, Coot and Refmac5 were used for solving the the structure by molecular replacement and refinement. Validation of the model was done using MolProbity. A summary of data collection and refinement data can be found in Table 1.

LC-MS data processing was performed as described in the "LC-MS Data Processing" section of the Method Details using Proteowizard, X!Tandem and trans-proteomic pipeline. Statistical analysis was done using SAINT to assign a Bayesian FDR.

The alignment of ULK4 kinase domain orthologs was performed using MAFFT and FastTree as described in the "Phylogenetic Tree" section of the Method Details.

Determination of the activation loop length of ULK4 orthologs was performed using MAPGAPS and R version as described in the "Activation Loop Length Distribution" section of the Method Details.

Molecular dynamics simulation of ULK4 was performed as described in the "Molecular Dynamics (MD) of ULK4 with ATP Bound" section of the Method Details using a variety of softwares listed in the Key Resources Table. For structural visualization PyMOL was used. 